JOURNAL OF

SYMPLECTIC GEOMETRY

Volume 2, Number 3, 309-355, 2004

\title{
SUBMANIFOLDS OF GENERALIZED COMPLEX MANIFOLDS
}

\author{
Oren Ben-Bassat and Mitya Boyarchenko
}

The main goal of our paper is the study of several classes of submanifolds of generalized complex manifolds. Along with the generalized complex submanifolds defined by Gualtieri and Hitchin in [Gua], [H3] (we call these "generalized Lagrangian submanifolds" in our paper), we introduce and study three other classes of submanifolds and their relationships. For generalized complex manifolds that arise from complex (resp., symplectic) manifolds, all three classes specialize to complex (resp., symplectic) submanifolds. In general, however, all three classes are distinct. We discuss some interesting features of our theory of submanifolds, and illustrate them with a few nontrivial examples. Along the way, we obtain a complete and explicit classification of all linear generalized complex structures.

We then support our "symplectic/Lagrangian viewpoint" on the submanifolds introduced in [Gua], [H3] by defining the "generalized complex category", modelled on the constructions of Guillemin-Sternberg [GS] and Weinstein [W2]. We argue that our approach may be useful for the quantization of generalized complex manifolds.

\section{Contents}

1. Introduction

2. Generalized complex linear algebra

3. Generalized complex subspaces and quotients 323

4. Split subspaces and the graph condition 327

5. Generalized complex manifolds and submanifolds 331

6. The generalized complex "category" 336

7. Examples and counterexamples 338 
8. Proofs

References

\section{Introduction}

1.1. Motivation. The goal of our paper is the study of certain special classes of submanifolds of generalized complex manifolds. The notion of a generalized complex manifold was introduced by Nigel Hitchin in [H2]. It contains as special cases both complex and symplectic manifolds. Later Marco Gualtieri and Hitchin ([Gua], [H3]) have defined a class of submanifolds of generalized complex manifolds which they have called "generalized complex submanifolds" and which in this paper we call "generalized Lagrangian submanifolds", for the reasons explained below. Hitchin's and Gualtieri's notion specializes to complex submanifolds of complex manifolds and to Lagrangian submanifolds of symplectic manifolds. In particular, if $M$ is a generalized complex manifold, then, in general, neither $M$ itself nor the points of $M$ are generalized complex submanifolds of $M$ in their terminology.

Our first task, then, was to find a different definition which gives complex submanifolds in the complex case and symplectic submanifolds in the symplectic case. It is also important to understand the relationship between our notion of a generalized complex submanifold and Hitchin and Gualtieri's notion; and, on the other hand, to try to see if Hitchin and Gualtieri's notion can be used in the theory of generalized complex manifolds in a similar way that Lagrangian submanifolds are used in symplectic geometry. For example, if $\phi: N \rightarrow M$ is a diffeomorphism between two symplectic manifolds, it is well-known (see, e.g., $[\mathbf{C d S}]$ ) that $\phi$ is a symplectomorphism if and only if the graph of $\phi$ is a Lagrangian submanifold of $N \times M$ with respect to the "twisted product symplectic structure" on $N \times M$. It is natural to ask if the obvious generalization of this result holds for generalized complex manifolds.

More generally, suppose $\left(M, \omega_{M}\right)$ is a symplectic manifold and $N$ is a submanifold of $M$ which carries a symplectic form $\omega_{N}$. Again, it is easy to see that $\omega_{N}=\left.\omega_{M}\right|_{N}$ if and only if the graph of the inclusion map $N \hookrightarrow M$ is an isotropic submanifold of $N \times M$ with respect to the twisted product symplectic structure. On the other hand, if $M$ is a real manifold, $N$ is a submanifold of $M$, and both $M$ and $N$ are equipped with complex structures, then $N$ is a complex submanifold of $M$ if and only if the graph of the inclusion map $N \hookrightarrow M$ is a complex submanifold of $N \times M$ with respect to the product complex structure.

A starting point for our discussion is the observation that the conditions appearing in Gualtieri and Hitchin's definition of a generalized complex submanifold can be relaxed naturally, so that the resulting object specializes 
to complex submanifolds in the complex case and to isotropic submanifolds in the symplectic case. We call these objects "generalized isotropic submanifolds." One of the main goals of our work was trying to generalize the remarks of the previous paragraph to find a relationship between generalized isotropic submanifolds and our notion of generalized complex submanifolds.

Another goal was to define and study the "generalized complex category", in the spirit of $[\mathbf{W 1}],[\mathbf{W 2}]$. The definition is based on the notion of a generalized Lagrangian submanifold. We believe that this "category" can be used to study quantization of generalized complex manifolds. (The word "category" appears in quotes, because, as explained in [W1] or [W2], a certain transversality condition has to be satisfied for a pair of morphisms to be composable.)

The class of submanifolds which we call generalized complex submanifolds is distinguished in that (in contrast to the others) its members naturally inherit a generalized complex manifold structure from the ambient manifold. After the first version of our paper has appeared we have learned of a new definition of a class of submanifolds of generalized complex manifolds which includes the submanifolds introduced by Gualtieri and Hitchin as a subclass. These submanifolds are called generalized complex branes in a recent paper of Kapustin [Kap] on Topological Strings. We do not investigate the relationships of generalized complex branes to the other submanifolds introduced in our paper.

1.2. Main results. Most of our paper is devoted to the study of generalized complex structures on real vector spaces. Only at the end do we apply our results to generalized complex manifolds. While we feel that our study of subspaces of generalized complex vector spaces is more or less complete, it is clear that our results for generalized complex manifolds have a preliminary nature. Still, we believe that the questions that we have raised are already fundamental at the vector space level and thus have to be addressed. Furthermore, as we show, the linear situation is far from being trivial (unlike in the theory of complex or symplectic vector spaces), due to the possibility of a $B$-field transformation. ${ }^{1}$ Some unexpected features of the theory are described below.

The first part of our paper is devoted to the study of induced generalized complex structures on subspaces of generalized complex vector spaces. The situation is quite similar to the one considered by Theodore Courant in [Cou]. In fact, many of our basic constructions are straightforward modifications of the ones introduced by Courant. However, there is an important difference: whereas a Dirac structure on a real vector space induces a Dirac

\footnotetext{
${ }^{1}$ These transformations, along with the $\beta$-field transformations, play a special role in the theory. They were first introduced in $[\mathbf{H 2}]$. We recall their definition in $\S 2.4$.
} 
structure on every subspace and quotient space, this is not so for generalized complex structures. Namely, recall that if $V$ is a finite-dimensional real vector space, then a generalized complex structure on $V$ can be defined by a subspace $E \subset V_{\mathbb{C}} \oplus V_{\mathbb{C}}^{*}$ which is isotropic with respect to the quadratic form $Q(v \oplus f)=-f(v)$ and satisfies $V_{\mathbb{C}} \oplus V_{\mathbb{C}}^{*}=E \oplus \bar{E}$, where the bar denotes the complex conjugate. Now, given a real subspace $W \subseteq V$, we can define, as in $[\mathbf{C o u}]$, the subspace $E_{W} \subset W_{\mathbb{C}} \oplus W_{\mathbb{C}}^{*}$ consisting of all pairs of the form $\left(w,\left.f\right|_{W_{\mathbb{C}}}\right)$, where $w \in W_{\mathbb{C}}$ and $f \in V_{\mathbb{C}}$ are such that $(w, f) \in E$. It is clear that $E_{W}$ is still isotropic with respect to $Q$. However, the condition $W_{\mathbb{C}} \oplus W_{\mathbb{C}}^{*}=E_{W} \oplus \bar{E}_{W}$ may no longer hold.

Thus we define $W$ to be a "generalized complex subspace" if the last condition does hold, so that we get an induced generalized complex structure. We then describe this induced structure in terms of spinors (Proposition 3.5). The dual notion, that of a quotient generalized complex structure, is also studied. We introduce an operation which interchanges generalized complex structures on a real vector space and on its dual; this operation can then be used to reduce many statements about quotients to statements about subspaces (and vice versa). Hence, naturally, we devote more attention to subspaces than to quotients. Since the original version of this paper was made available we have realized that this duality operation corresponds to the idea of T-duality from the physics literature, see $[\mathbf{K O}, \mathbf{T W}]$ and the references therein. This idea is used to establish a form of mirror symmetry for generalized complex manifolds in a recent preprint $[\mathbf{B}]$ by the first author. We also prove the classification theorem for generalized complex vector spaces: every generalized complex structure is the $B$-field transform of a direct sum of a complex structure and a symplectic structure (Theorem 3.10). This result has applications to the study of constant generalized complex structures on tori, both with regards to quantization and Morita equivalence (cf. $[\mathbf{T W}]$ ), and mirror symmetry (cf. $[\mathbf{K O}]$ ).

At this point we should mention that there is some overlap between our paper and Gualtieri's thesis [Gua]. Most notably, this applies to our classification theorem mentioned above, which corresponds to Theorem 4.13 in [Gua]. However, the first version of our paper was placed on the e-Print archive and submitted for publication before [Gua] became available to (us and) the general audience; in particular, we have obtained the classification theorem independently. Of course, many definitions given in our paper can be found in [Gua]; they are given here for completeness, and our paper is self-contained and can be read independently of [Gua].

Let us emphasize that there are several subtle points in the theory of subspaces that we develop, which are not present in the theory of complex or symplectic structures on vector spaces. For example, if $W$ is a generalized complex subspace of a generalized complex vector space $V$, then $V / W$ need 
not be a generalized complex quotient of $V$ (cf. Example 7.6). As another example of "strange" behavior, we show the following. If $V$ is any generalized complex vector space, there is a unique maximal subspace $S$ of $V$ such that $S$ is a GC subspace and the induced structure on $S$ is $B$-symplectic (Theorem 3.11). [Hereafter, we use the expressions " $B$-symplectic structure" and " $B$-complex structure" as shorthand for " $B$-field transform of a symplectic structure" and " $B$-field transform of a complex structure," respectively.] Furthermore, there is a canonical complex structure $J$ on the quotient $V / S$. However, $V / S$ may not be a generalized complex quotient of $V$, in the sense of Definition 3.8. (See Example 7.7.) On the other hand, if $V / S$ is a generalized complex quotient of $V$, then the induced structure on $V / S$ is $B$-complex, and the associated complex structure coincides with $J$.

The interplay between $B$-field transforms and $\beta$-field transforms is also delicate. For instance, if a generalized complex structure is both a $B$-field transform and a $\beta$-field transform of a (classical) complex structure, then it is, in fact, complex. The corresponding statement for symplectic structures is false. On the other hand, it is possible to start with a classical symplectic structure, make a $B$-field transform, then a $\beta$-field transform, and arrive at a classical complex structure. These features are discussed in some detail in $\S 7.4$.

To describe the results of the second part of the paper, we need to introduce more notation. Let $V$ be as above, and recall that another way to describe a generalized complex structure on $V$ is by giving an automorphism $\mathcal{J}_{V}$ of $V \oplus V^{*}$ which satisfies $\mathcal{J}_{V}^{2}=-1$ and preserves the quadratic form $Q$ on $V \oplus V^{*}$. [An equivalence with the previous definition is obtained by assigning to such a $\mathcal{J}_{V}$ its $+i$-eigenspace $E$ in $V_{\mathbb{C}} \oplus V_{\mathbb{C}}^{*}$.] For many considerations it is convenient to write $\mathcal{J}_{V}$ in matrix form:

$$
\mathcal{J}_{V}=\left(\begin{array}{cc}
\mathcal{J}_{1} & \mathcal{J}_{2} \\
\mathcal{J}_{3} & \mathcal{J}_{4}
\end{array}\right)
$$

where $\mathcal{J}_{1}: V \rightarrow V, \mathcal{J}_{2}: V^{*} \rightarrow V, \mathcal{J}_{3}: V \rightarrow V^{*}$ and $\mathcal{J}_{4}: V^{*} \rightarrow V^{*}$ are linear maps.

Let $W$ be a real subspace of $V$. We call $W$ a generalized isotropic (resp. generalized coisotropic, resp. generalized Lagrangian) subspace of $V$ if $\mathcal{J}_{V}(W) \subseteq W \oplus \operatorname{Ann}(W)$ (resp. $\mathcal{J}_{V}(\operatorname{Ann}(W)) \subseteq W \oplus \operatorname{Ann}(W)$, resp. $W \oplus \operatorname{Ann}(W)$ is stable under $\left.\mathcal{J}_{V}\right)$. We say that $W$ is split if there exists a subspace $N \subseteq V$ such that $V=W \oplus N$ and $W \oplus \operatorname{Ann}(N) \subseteq V \oplus V^{*}$ is stable under $\mathcal{J}$. [In fact, this is equivalent to the condition that there is a decomposition $V=W \oplus N$ as a direct sum of generalized complex structures, which explains the terminology.] 
On the other hand, we define the "opposite generalized complex structure" on $V$ to be the one corresponding to the automorphism

$$
\tilde{\mathcal{J}}_{V}=\left(\begin{array}{cc}
\mathcal{J}_{1} & -\mathcal{J}_{2} \\
-\mathcal{J}_{3} & \mathcal{J}_{4}
\end{array}\right)
$$

If $W$ is equipped with some generalized complex structure $\mathcal{J}_{W}$, we say that this subspace satisfies the graph condition (with respect to $\mathcal{J}_{W}$ ) if the graph of the inclusion map $W \hookrightarrow V$ is a generalized isotropic subspace of $W \oplus$ $V$ with respect to the "twisted product structure" on $W \oplus V$, which by definition is the direct sum of $\tilde{\mathcal{J}}_{W}$ and $\mathcal{J}_{V}$.

The following three results (see $\$ 4.4$ ) explain the relations between the three types of subspaces of a generalized complex vector space $V$ that we have introduced.

Theorem 1. Every split subspace is a generalized complex subspace. Furthermore, it satisfies the graph condition with respect to the induced generalized complex structure.

Theorem 2. A generalized complex subspace $W \subseteq V$ satisfies the graph condition with respect to the induced structure if and only if $W$ is invariant under $\mathcal{J}_{1}$.

It is worth emphasizing that if $W$ is a generalized complex subspace and it satisfies the graph condition with respect to some generalized complex structure, this structure does not necessarily have to be the induced one. More precisely:

Theorem 3. Suppose that $W \subseteq V$ is an arbitrary subspace and $\mathcal{J}_{W}$ is a generalized complex structure on $W$ such that the graph condition is satisfied. If $W$ is a generalized complex subspace, then the graph condition is also satisfied with respect to the induced generalized complex structure, and $\mathcal{J}_{W}$ is a $\beta$-field transform of the induced structure on $W$.

In Remark 7.4, we show that a subspace satisfying the graph condition with respect to some generalized complex structure does not have to be a generalized complex subspace in our sense. This may be seen as evidence of the fact that our definition of the graph condition is too weak. One possible explanation for this is that, unlike the classical symplectic situation, a generalized isotropic subspace which has half the dimension of the ambient space is not necessarily generalized Lagrangian. Thus, for example, if $\mu$ : $V \rightarrow W$ is an isomorphism of real vector spaces, and $V, W$ are equipped with generalized complex structures such that the graph of $\mu$ is generalized isotropic with respect to the twisted product structure, then it does not necessarily follow that $\mu$ induces an isomorphism between the two structures. On the other hand, we have the following 
Theorem 4. Let $V, W$ be generalized complex vector spaces, and let $\mu$ : $V \rightarrow W$ be an isomorphism of the underlying real vector spaces. If the graph of $\mu$ is generalized Lagrangian with respect to the twisted product structure on $V \oplus W$, then $\mu$ induces an isomorphism between the two generalized complex structures.

The global version of this result is given in Theorem 6.1.

In the second part of the paper we generalize all the notions introduced previously to generalized complex manifolds. In addition, we study the analogue of the notion of an "admissible function" introduced by Courant [Cou]. This leads to the definition of the Courant sheaf: if $M$ is a generalized complex manifold, with $\mathcal{J}_{M}$ the corresponding automorphism of $T M \oplus T^{*} M$ and $\bar{E}$ the $-i$ eigenbundle of $\mathcal{J}_{M}$ on $T_{\mathbb{C}} M \oplus T_{\mathbb{C}}^{*} M$, the sections of the Courant sheaf are pairs $(f, X)$, where $f$ is a complex-valued $C^{\infty}$ function and $X$ is a section of $T_{\mathbb{C}} M$ such that $d f+X \in \bar{E}$. We prove that the Courant sheaf is a sheaf of local rings and also a sheaf of Poisson algebras. Moreover, for a split submanifold $N$ of a generalized complex manifold $M$, we define a natural operation of "pullback" of sections of the Courant sheaf on $M$ to those on $N$ (Theorem 5.13). The generalized complex structure on $M$ can be recovered from the Courant sheaf together with its embedding into $C_{M}^{\infty} \oplus T_{\mathbb{C}} M$ in case $M$ is complex or symplectic, but it seems that, in general, additional information is required.

The last part of the paper is devoted to the generalized complex "category". Recall that Weinstein [W2] has introduced the symplectic "category", based on some ideas of Guillemin and Sternberg [GS]. The objects of this "category" are symplectic manifolds. If $M$ and $N$ are two symplectic manifolds, the set of morphisms from $M$ to $N$ is defined to be the set of submanifolds of $M \times N$ that are Lagrangian with respect to the "twisted product symplectic structure" on $M \times N$. Two morphisms, $M \rightarrow N$ and $N \rightarrow K$, can be composed provided a certain transversality condition is satisfied (see [W2] for details). In $\S 6.2$ we generalize Weinstein's construction by replacing symplectic manifolds with generalized complex manifolds, and Lagrangian submanifolds with generalized Lagrangian submanifolds. In the linear case $(\S 6.3)$, where the transversality condition disappears, we get an honest category, thereby generalizing a construction of Guillemin and Sternberg [GS]. We would like to point out that one of the applications of Guillemin-Sternberg-Weinstein construction is to deformation quantization and geometric quantization of symplectic manifolds (see [GS], where the quantization problem is solved completely in the linear case, and [W1], [W2] for a discussion of the global case). We hope that, similarly, the constructions of Section 6 can be used for quantization of generalized complex structures. We plan to study this in detail in subsequent publications. 
1.3. Structure of the paper. Our paper is organized as follows. In Section 2 we recall the basic definitions of the theory of generalized complex vector spaces. We follow $[\mathbf{G u a}]$ and $[\mathbf{H 3}]$ for the most part. We also list the basic definitions and results from the theory of spinors, referring to the book $[\mathbf{C}]$ for proofs. We then define an operation which interchanges generalized complex structures on a real vector space with those on its dual, and we conclude the section with a discussion of $B$-field and $\beta$-field transforms. In Section 3 we introduce and study the notions of "generalized complex subspaces" (by modifying the construction of [Cou]) and "generalized complex quotients." We also prove the classification theorem for generalized complex structures, as well as certain weaker but more "canonical" analogues of the theorem; a consequence of our theory is that every generalized complex vector space has even dimension over $\mathbb{R}$. In Section 4 we introduce the notions of "split subspaces" and "subspaces satisfying the graph condition" and describe in detail the relationship of these notions with the more general notion of a generalized complex subspace.

In Section 5 we recall the definitions of generalized almost complex manifolds and generalized complex manifolds. For the latter, we study the natural generalization of the notion of "admissible function" defined by Courant, and we introduce the "Courant sheaf." We then define the global analogues of generalized complex subspaces and split subspaces: "generalized complex submanifolds" and "split submanifolds" of generalized complex manifolds. We end the section by defining the "pullback" of sections of the Courant sheaf on a generalized complex manifold to a split submanifold. In Section 6 , we study the "graph condition" for submanifolds of generalized complex manifolds. This idea allows us to define the "generalized complex category" à la Weinstein [W2]. We finish by studying the simpler category of generalized complex vector spaces, modelled on the construction of Guillemin and Sternberg $[\mathbf{G S}]$.

In Section 7 we illustrate our main constructions and results with two types of examples: $B$-field transforms of complex and symplectic structures. (A few scattered examples also appear throughout the text.) More specifically, we characterize the GC subspaces (resp. subspaces satisfying the graph condition, resp. split subspaces) of $B$-complex and $B$-symplectic vector spaces, we characterize generalized isotropic (resp. coisotropic, resp. Lagrangian) subspaces of complex and symplectic vector spaces, and we state Gualtieri's characterization of generalized Lagrangian submanifolds of $B$-complex and $B$-symplectic manifolds. We then explain some interesting features of $B$-field transforms of symplectic manifolds. Finally, we discuss in detail some nontrivial counterexamples that we mentioned in $\S 1.2$.

We have only included the proofs of the most trivial results in the main body of the paper. All other proofs appear in a separate section (Section 
8) at the end of the article. This clarifies the exposition, and allows us to present our results in not necessarily the same order in which they are proved.

For the reader's convenience, a more detailed summary appears at the beginning of each section.

1.4. Acknowledgements. We are grateful to Marco Gualtieri for sharing some of his notes with us, prior to the publication of his thesis. The content of those notes was used in this paper via several of the definitions and constructions of Sections 2, 5 and 7 as is noted in the appropriate places. We would also like to thank Tony Pantev for the suggestion to work on generalized complex manifolds, as well as for many useful comments on our paper and suggestions for improvement. Last but not least, we are indebted to our referees for suggesting several essential improvements to the style of our presentation and pointing out the missing references.

1.5. Notation and conventions. All vector spaces considered in this paper will be finite-dimensional. We will be dealing with both real and complex vector spaces. If $V$ is a real vector space, we denote by $V_{\mathbb{C}}$ its complexification. If $W \subseteq V$ is a real subspace, we will identify $W_{\mathbb{C}}$ with a subspace of $V_{\mathbb{C}}$ in the usual way. The complex conjugation on $\mathbb{C}$ extends to an $\mathbb{R}$-linear automorphism of $V_{\mathbb{C}}$ which we denote by $x \mapsto \bar{x}$. Recall that a complex subspace $U$ of $V_{\mathbb{C}}$ is of the form $W_{\mathbb{C}}$ for some real subspace $W \subseteq V$ if and only if $U=\bar{U}$.

The dual of a real or complex vector space $V$ will be denoted by $V^{*}$. We will always implicitly identify $V$ with $\left(V^{*}\right)^{*}$. If $W \subseteq V$ is a real (resp., complex) subspace, we write $\operatorname{Ann}(W)=\left\{f \in V^{*}|f|_{W} \equiv 0\right\}$. Similarly, if $U \subseteq V^{*}$ is a real or complex subspace, we write $\operatorname{Ann}(U)=\{v \in V \mid f(v)=$ $0 \forall f \in U\}$.

If $V$ is real, we identify $\left(V_{\mathbb{C}}\right)^{*}$ with $\left(V^{*}\right)_{\mathbb{C}}$ in the natural way. The natural projections $V \oplus V^{*} \rightarrow V$ and $V \oplus V^{*} \rightarrow V^{*}$, or their complexifications, will be denoted by $\rho$ and $\rho^{*}$, respectively. We have the standard indefinite nondegenerate symmetric bilinear pairing $\langle\cdot, \cdot\rangle$ on $V \oplus V^{*}$, given by ${ }^{2}$

$$
\langle v+f, w+g\rangle=-\frac{1}{2}(f(w)+g(v)) \text { for all } v, w \in V, f, g \in V^{*} .
$$

We use the same notation for the complexification of $\langle\cdot, \cdot\rangle$. Usually, if $v \in V$ and $f \in V^{*}$, we will write either $\langle f \mid v\rangle$ or $\langle v \mid f\rangle$ for $f(v)$. The pairing $\langle\cdot, \cdot\rangle$ corresponds to the quadratic form $Q(v+f)=-f(v)$.

The exterior algebra of a vector space $V$ will be denoted by $\wedge^{\bullet} V$. If $x \in V$ and $\xi \in \Lambda^{\bullet} V^{*}$, we write $\iota_{x} \xi$ for the contraction of $x$ with $\xi$. An element $B \in \bigwedge^{2} V^{*}$ will be identified with the linear map $V \rightarrow V^{*}$ that it

\footnotetext{
${ }^{2}$ Our convention here follows that of $[\mathbf{H 3}]$.
} 
induces; similarly, an element $\beta \in \bigwedge^{2} V$ will be identified with the induced linear map $V^{*} \rightarrow V$. We will frequently consider endomorphisms of $V \oplus V^{*}$. These will usually be written as $2 \times 2$ matrices

$$
T=\left(\begin{array}{ll}
T_{1} & T_{2} \\
T_{3} & T_{4}
\end{array}\right)
$$

with the understanding that $T_{1}: V \rightarrow V, T_{2}: V^{*} \rightarrow V, T_{3}: V \rightarrow V^{*}$ and $T_{4}: V^{*} \rightarrow V^{*}$ are linear maps. In this situation, elements of $V \oplus V^{*}$ will be written as column vectors: $(v, f)^{t}$, where $v \in V, f \in V^{*}$.

For us, a real (resp., complex) manifold will always mean a $C^{\infty}$ real (resp., complex-analytic) finite-dimensional manifold. If $M$ is a real manifold, by $C_{M}^{\infty}$ we denote the sheaf of (germs of) complex-valued $C^{\infty}$ functions on $M$. We will denote by $T M$ and $T^{*} M$ the tangent and cotangent bundles of $M$, and by $T_{\mathbb{C}} M$ and $T_{\mathbb{C}}^{*} M$ their complexifications. The pairing $\langle\cdot, \cdot\rangle$ defined above at the level of vector spaces extends immediately to a pairing $\langle\cdot, \cdot\rangle: V_{\mathbb{C}} \times V_{\mathbb{C}}^{*} \rightarrow C_{M}^{\infty}$, where $V$ is any vector bundle on $M$ and $V_{\mathbb{C}}$ is its complexification. The de Rham differential on $\wedge^{\bullet} T_{\mathbb{C}}^{*} M$ will be denoted by $\omega \mapsto d \omega$. For a section $X$ of $T_{\mathbb{C}} M$, we will denote by $\iota_{X}$ and $\mathcal{L}_{X}$ the operators of contraction with $X$ and the Lie derivative in the direction of $X$, which are $\mathbb{C}$-linear operators on $\Lambda^{\bullet} T_{\mathbb{C}}^{*} M$.

By a complex structure on a real vector space $V$ we mean an $\mathbb{R}$-linear automorphism $J$ of $V$ satisfying $J^{2}=-1$. (Other authors call such a $J$ an almost complex structure.) The following abbreviations will be frequently used in our paper: $\mathrm{GC}=$ "generalized complex", $\mathrm{GAC}=$ "generalized almost complex", GCS= "GC structure", GACS="GAC structure", GCM="generalized complex manifold", GACM="generalized almost complex manifold".

\section{Generalized complex linear algebra}

2.1. Equivalent definitions. We begin by giving three ways of defining a GCS on a real vector space, and then showing their equivalence. We will see later that in various contexts, one of these descriptions may be better than the others, but there is no description that is "the best" universally. Thus we prefer to work with all of them interchangeably.

Proposition 2.1. Let $V$ be a real vector space. There are natural bijections between the following 3 types of structure on $V$.

(1) $A$ subspace $E \subset V_{\mathbb{C}} \oplus V_{\mathbb{C}}^{*}$ such that $E \cap \bar{E}=(0)$ and $E$ is maximally isotropic with respect to the standard pairing $\langle\cdot, \cdot\rangle$ on $V_{\mathbb{C}} \oplus V_{\mathbb{C}}^{*}$.

(2) An $\mathbb{R}$-linear automorphism $\mathcal{J}$ of $V \oplus V^{*}$ such that $\mathcal{J}^{2}=-1$ and $\mathcal{J}$ is orthogonal with respect to $\langle\cdot, \cdot\rangle$.

(3) A pure spinor $\phi \in \bigwedge^{\bullet} V_{\mathbb{C}}^{*}$, defined up to multiplication by a nonzero scalar, such that $\langle\phi, \bar{\phi}\rangle_{M} \neq 0$, where $\langle\cdot, \cdot\rangle_{M}$ denotes the Mukai pairing $([\mathbf{C}],[\mathbf{H 2}],[\mathbf{H 3}])$. 
Remark 2.2. Our notation is different from the one of Gualtieri [Gua], who uses $L \subset V_{\mathbb{C}} \oplus V_{\mathbb{C}}^{*}$ to denote a generalized complex structure, and $E$ the projection of the subspace $L$ onto $V_{\mathbb{C}}$.

The terminology (3) is explained in $\S 2.2$ below, and the bijections are made explicit in $\S 8.1$. A structure of one of these types will be called a generalized complex structure on $V$, and a real vector space equipped with a GC structure will be called a generalized complex vector space.

Remark 2.3. In $[\mathbf{H 1}],[\mathbf{H 2}]$ it is assumed from the very beginning that generalized complex manifolds have even dimension (as real manifolds). The reader may also assume that our GC vector spaces have even real dimension; this is used in an essential way in the arguments that involve spinors (cf. Theorem 2.5(2.5)). However, we can prove that, with the definitions of a GC structure on $V$ in terms of $E \subset V_{\mathbb{C}} \oplus V_{\mathbb{C}}^{*}$ or $\mathcal{J} \in \operatorname{Aut}_{\mathbb{R}}\left(V \oplus V^{*}\right)$, the even-dimensionality of $V$ is automatic (Corollary 3.12). We invite the reader to check that our proof of Corollary 3.12 does not depend on any statements about spinors. The result was also proved in [H3] by a different method (by showing that a GC structure on a real manifold induces an almost complex structure).

2.2. Recollection of spinors. Here we list the basic definitions and results from the theory of spinors, following $[\mathbf{C}]$ (and, in a few places, $[\mathbf{H 3}]$ ), that will be used in the sequel. Chevalley's description of the theory is somewhat more general than presented below; however, the reader should have no difficulty modifying the notation and results of $[\mathbf{C}]$ to fit our situation.

Let $V$ be a real vector space, and write $V^{d}=V \oplus V^{*}$. Recall the quadratic form $Q(v+f)=-\langle v \mid f\rangle=-\iota_{v}(f)$ on $V^{d}$. We let Cliff $(V)$ denote the Clifford algebra of the complexification $V_{\mathbb{C}}^{d}$ corresponding to the quadratic form $Q$. This means (see $[\mathbf{C}]$ ) that $\operatorname{Cliff}(V)$ is the quotient of the tensor algebra on $V_{\mathbb{C}}^{d}$ by the ideal generated by all expressions of the form $e \otimes e-Q(e), e \in V_{\mathbb{C}}^{d}$. Note that if $E$ is any isotropic (w.r.t. $Q$ ) subspace of $V_{\mathbb{C}}^{d}$, then the subalgebra of $\operatorname{Cliff}(V)$ generated by $E$ is naturally isomorphic to the exterior algebra $\bigwedge^{\bullet} E$. In particular, $\operatorname{Cliff}(V)$ contains $S(V):=\bigwedge^{\bullet} V_{\mathbb{C}}^{*}$ in a natural way. We call $S(V)$ the space of spinors. The subspaces of $S(V)$ spanned by forms of even and odd degree are denoted by $S_{+}(V)$ and $S_{-}(V)$, respectively. We call $S_{+}(V)$ (resp., $S_{-}(V)$ ) the space of even (resp., odd) half-spinors.

There exists a representation of $\operatorname{Cliff}(V)$ on $S(V)$, with the action of the generating subspace $V_{\mathbb{C}}^{d} \subset \operatorname{Cliff}(V)$ being defined by

$$
(v+f) \cdot \phi=\iota_{v} \phi+f \wedge \phi, \quad v \in V_{\mathbb{C}}, f \in V_{\mathbb{C}}^{*}, \phi \in S(V) .
$$

Let $\phi \in S(V), \phi \neq 0$, and let $E$ be the annihilator of $\phi$ in $V_{\mathbb{C}}^{d}$, with respect to the action defined above. Observe that if $e \in E$, then $0=e \cdot e \cdot \phi=Q(e) \cdot \phi$, whence $Q(e)=0$. Thus $\left.Q\right|_{E} \equiv 0$, so $E$ is isotropic with respect to $Q$. 
Definition 2.4. We say that $\phi$ is a pure spinor if $E$ is maximally isotropic, i.e., $\operatorname{dim}_{\mathbb{C}} E=\operatorname{dim}_{\mathbb{R}} V$. In this case, we also call $\phi$ a representative spinor for $E$.

Recall from $\S 1.5$ that complex conjugation acts on $V_{\mathbb{C}}^{d}$ and on $S(V)$. It is clear that $\phi \in S(V)$ is a pure spinor if and only if $\bar{\phi}$ is such; and if $\phi$ is a representative spinor for a maximally isotropic subspace $E \subset V_{\mathbb{C}}^{d}$, then $\bar{\phi}$ is a representative spinor for $\bar{E}$, and vice versa.

The last ingredient that we need is the Mukai pairing $\langle\cdot, \cdot\rangle_{M}: S(V) \times$ $S(V) \rightarrow \bigwedge^{n} V$, where $n=\operatorname{dim}_{\mathbb{R}} V$ (see [C], §III.3.2; [H2], [H3]). However, we have decided to omit the definition of this pairing, since it is described in detail in $[\mathbf{C}]$ and $[\mathbf{H 3}]$. The only fact that we need about this pairing is Theorem 2.5(2.5), which is proved in [H3]. We refer the reader to [H3] for a detailed discussion of the Mukai pairing that is most relevant to our situation.

We can now state the main results from the theory of spinors that we will use in the paper.

\section{Theorem 2.5.}

(a) Every pure spinor is either even or odd: if $\phi \in S(V)$ is pure, then $\phi \in S_{+}(V)$ or $\phi \in S_{-}(V)$.

(b) Let $E \subset V_{\mathbb{C}}^{d}$ be a maximally isotropic subspace; then the space

$$
A_{E}=\{\phi \in S(V) \mid e \cdot \phi=0 \forall e \in E\}
$$

is one dimensional. Thus the nonzero elements of $A_{E}$ are precisely the representative spinors for $E$. In other words, we have a bijective correspondence between maximally isotropic subspaces of $V_{\mathbb{C}}^{d}$ and pure spinors modulo nonzero scalars.

(c) Let $E, F \subset V_{\mathbb{C}}^{d}$ be maximally isotropic subspaces corresponding to pure spinors $\phi, \psi \in S_{ \pm}(V)$. Then $E \cap F=(0)$ if and only if $\langle\phi, \psi\rangle_{M} \neq 0$.

(d) In particular, if $E \subset V_{\mathbb{C}}^{d}$ is a maximally isotropic subspace corresponding to a pure spinor $\phi$, then $E \cap \bar{E}=(0)$ if and only if $\langle\phi, \bar{\phi}\rangle_{M} \neq 0$.

(e) A spinor $\phi \in S(V)$ is pure if and only if it can be written as

$$
\phi=c \cdot \exp (u) \wedge f_{1} \wedge \cdots \wedge f_{k},
$$

where $c \in \mathbb{C}^{\times}, u \in \bigwedge^{2} V^{*}$, and $f_{1}, \ldots, f_{k}$ are linearly independent elements of $V_{\mathbb{C}}^{*}$. If $\phi$ is representative for a maximally isotropic subspace $E \subset V_{\mathbb{C}}^{d}$, then $f_{1}, \ldots, f_{k}$ form a basis for $E \cap V_{\mathbb{C}}^{*}$.

(f) Suppose that $\operatorname{dim}_{\mathbb{R}} V$ is even, and let $\phi \in S(V)$ be a pure spinor written in the form (2.1). Then, up to a nonzero scalar multiple, we have

$$
\langle\phi, \bar{\phi}\rangle_{M}=(u-\bar{u})^{p} \wedge f_{1} \wedge \cdots \wedge f_{k} \wedge \bar{f}_{1} \wedge \cdots \wedge \bar{f}_{k},
$$

where $p=n / 2-k$. 
Proofs. (a) [C], III.1.5. (b) The proof is contained in $[\mathbf{C}]$, §III.1. (c) $[\mathbf{C}]$, III.2.4. (d) This is immediate from (c). (e) [C], III.1.9. (f) See [H3].

2.3. Duality. Given a real vector space $V$, let $\tau: V \oplus V^{*} \rightarrow V^{*} \oplus V$ be the transposition of the two summands. Recalling the natural identification of $V^{* *}$ with $V$, we can also view $\tau$ as an isomorphism between $V \oplus V^{*}$ and $V^{*} \oplus V^{* *}$. We will continue to denote by $\tau$ the induced isomorphism $V_{\mathbb{C}} \oplus V_{\mathbb{C}}^{*} \rightarrow V_{\mathbb{C}}^{*} \oplus V_{\mathbb{C}}^{* *} \cong V_{\mathbb{C}}^{*} \oplus V_{\mathbb{C}}$. This duality operation corresponds to the idea from the physics literature of T-duality, see $[\mathbf{K O}, \mathbf{T W}]$ and the references therein. An explicit formula for the spinor representing the dual GC structure on $V^{*}$ in terms of the spinor representing the GC structure on $V$ turns out to be a Fourier-Mukai type of transformation, as in explained in $[\mathbf{B}]$ in relationship to a version of mirror symmetry for generalized complex manifolds.

Proposition 2.6. The isomorphism $\tau$ induces a bijection between $G C$ structures on $V$ and $G C$ structures on $V^{*}$. Explicitly, $E \subset V_{\mathbb{C}} \oplus V_{\mathbb{C}}^{*}$ is a $G C S$ on $V$ if and only if $\tau(E)$ is a GCS on $V^{*}$. If $E$ corresponds to $\mathcal{J} \in \operatorname{Aut}_{\mathbb{R}}\left(V \oplus V^{*}\right)$ as in Proposition 2.1, then $\tau(E)$ corresponds to $\tau \circ \mathcal{J} \circ \tau^{-1}$.

Proof. If is clear that $\tau$ preserves the form $\langle\cdot, \cdot\rangle$, and if $E \subseteq V_{\mathbb{C}} \oplus V_{\mathbb{C}}^{*}$ is any subspace, then $\tau(\bar{E})=\overline{\tau(E)}$. The proposition follows trivially from these observations.

2.4. $B$ - and $\beta$-field transforms. Let $V$ be a real vector space and $B \in$ $\bigwedge^{2} V^{*}$. We form the matrix (see $\left.\S 1.5\right)$

$$
\mathcal{B}:=\left(\begin{array}{cc}
1 & 0 \\
B & 1
\end{array}\right)
$$

It is straightforward to verify that $\mathcal{B}$ is an orthogonal automorphism of $V \oplus V^{*}$. Thus, if $E$ is a GCS on $V$, then $\mathcal{B} \cdot E$ is another one. We call $\mathcal{B} \cdot E$ the $B$-field transform of $E$ defined by $B$.

Remark 2.7. The notion of a "gauge transformation" for Dirac structures has been introduced and studied by Severa and Weinstein in $[\mathbf{S W}], \S 3$. The obvious complex analogue of this notion gives rise to the $B$-field transforms that we have just defined.

Similarly, if $\beta \in \bigwedge^{2} V$, then the matrix

$$
\mathcal{B}^{\prime}:=\left(\begin{array}{cc}
1 & \beta \\
0 & 1
\end{array}\right)
$$

also acts on GC structures on $V$; if $E$ is one, then $\mathcal{B}^{\prime} \cdot E$ will be called the $\beta$-field transform of $E$ defined by $\beta$. If we look at GC structures in terms of the corresponding orthogonal automorphisms $\mathcal{J}$ of $V \oplus V^{*}$, then the actions of $B$ and $\beta$ are given by $\mathcal{J} \mapsto \mathcal{B} \cdot \mathcal{J} \cdot \mathcal{B}^{-1}$ and $\mathcal{J} \mapsto \mathcal{B}^{\prime} \cdot \mathcal{J} \cdot \mathcal{B}^{\prime-1}$, respectively. We can also describe $B$-field transforms in terms of spinors: 
Proposition 2.8. Suppose that a GCS on a real vector space $V$ is defined by a pure spinor $\phi \in \bigwedge^{\bullet} V_{\mathbb{C}}^{*}$, and let $B \in \bigwedge^{2} V^{*}$. Then the $B$-field transform of this structure corresponds to the pure spinor $\exp (-B) \wedge \phi$.

We leave to the reader the task of describing $\beta$-field transforms in a similar way.

Remark 2.9. Suppose that $E$ is a GCS on a real vector space $V$ and $E^{\prime}$ is the $B$-field transform of $E$ defined by $B \in \bigwedge^{2} V^{*}$. Then, obviously, $\tau\left(E^{\prime}\right)$ is the $\beta$-field transform of $\tau(E)$, defined by the same $B \in \bigwedge^{2} V^{*}$ (but viewed now as a bivector on $V^{*}$ ). Thus, the operation $\tau$ interchanges $B$ - and $\beta$-field transforms.

Definition 2.10. Let $V$ be a real vector space.

(1) Let $J$ be a (usual) complex structure on $V$. Then

$$
\mathcal{J}=\left(\begin{array}{cc}
J & 0 \\
0 & -J^{*}
\end{array}\right)
$$

is a GC structure on $V$. If $\mathcal{J}$ is a GCS on $V$ that can be written in this form, we say that $\mathcal{J}$ is complex.

(2) A $B$-field (resp., $\beta$-field) transform of a complex GCS on $V$ will be referred to as a $B$-complex (resp., $\beta$-complex) structure on $V$.

(3) Let $\omega$ be a symplectic form on $V$ (i.e., a nondegenerate form $\omega \in \wedge^{2} V^{*}$ ). Then

$$
\mathcal{J}=\left(\begin{array}{cc}
0 & -\omega^{-1} \\
\omega & 0
\end{array}\right)
$$

is a GC structure on $V$. If $\mathcal{J}$ is a GCS on $V$ that can be written in this form, we say that $\mathcal{J}$ is symplectic.

(4) A $B$-field (resp., $\beta$-field) transform of a symplectic GCS on $V$ will be referred to as a $B$-symplectic (resp., $\beta$-symplectic) structure on $V$.

Proposition 2.11. Let $V$ be a real vector space and $E \subset V_{\mathbb{C}} \oplus V_{\mathbb{C}}^{*}$ a $G C S$ on $V$. Write

$$
\mathcal{J}=\left(\begin{array}{cc}
\mathcal{J}_{1} & \mathcal{J}_{2} \\
\mathcal{J}_{3} & \mathcal{J}_{4}
\end{array}\right)
$$

for the corresponding orthogonal automorphism of $V \oplus V^{*}$. Then

(a) $E$ is B-complex (resp., $\beta$-complex) $\Longleftrightarrow \rho(E) \cap \rho(\bar{E})=(0)$ (resp., $\left.\rho^{*}(E) \cap \rho^{*}(\bar{E})=(0)\right) \Longleftrightarrow \mathcal{J}_{2}=0$ (resp., $\left.\mathcal{J}_{3}=0\right) \Longleftrightarrow V_{\mathbb{C}}^{*}=$ $\left(V_{\mathbb{C}}^{*} \cap E\right)+\left(V_{\mathbb{C}}^{*} \cap \bar{E}\right)\left(\right.$ resp.,$\left.V_{\mathbb{C}}=\left(V_{\mathbb{C}} \cap E\right)+\left(V_{\mathbb{C}} \cap \bar{E}\right)\right) ;$

(b) $E$ is complex if and only if it is both $B$-complex and $\beta$-complex;

(c) $E$ is B-symplectic (resp., $\beta$-symplectic) $\Longleftrightarrow E \cap V_{\mathbb{C}}^{*}=(0)$ (resp., $E \cap$ $\left.V_{\mathbb{C}}=(0)\right) \Longleftrightarrow \mathcal{J}_{2}$ is an isomorphism (resp., $\mathcal{J}_{3}$ is an isomorphism) $\Longleftrightarrow \rho(E)=V_{\mathbb{C}}\left(\right.$ resp., $\left.\rho^{*}(E)=V_{\mathbb{C}}^{*}\right)$;

(d) $E$ is symplectic if and only if $\mathcal{J}_{1}=0$. 
The analogue of part (b) fails in the symplectic case, as we will see in $\S 7.4$.

\section{Generalized complex subspaces and quotients}

3.1. Definition of GC subspaces. Let $V$ be a real vector space with a GC structure $E \subset V_{\mathbb{C}} \oplus V_{\mathbb{C}}^{*}$. Given a subspace $W \subseteq V$, we define (following [Cou])

$$
E_{W}=\left\{\left(\rho(e),\left.\rho^{*}(e)\right|_{W_{\mathbb{C}}}\right) \mid e \in E \cap\left(W_{\mathbb{C}} \oplus V_{\mathbb{C}}^{*}\right)\right\} .
$$

Clearly, $E_{W}$ is an isotropic subspace of $W_{\mathbb{C}} \oplus W_{\mathbb{C}}^{*}$. It is straightforward to compute that $\operatorname{dim}_{\mathbb{C}} E_{W}=\operatorname{dim}_{\mathbb{R}} W$ (see Lemma 8.2); thus $E_{W}$ is, in fact, maximally isotropic.

Remark 3.1. The definition of $E_{W}$ and the fact that it is maximally isotropic can be obtained by specializing the much more general discussion of the functorial properties of linear Dirac structures, cf. [BR]. We have included the proof in our paper for the sake of completeness.

Definition 3.2. We say that $W$ is a generalized complex subspace of $V$ if $E_{W} \cap \bar{E}_{W}=(0)$, so that $E_{W}$ is a generalized complex structure on $W$. In this case, we denote by $\mathcal{J}_{W}$ the orthogonal automorphism of $W \oplus W^{*}$ corresponding to $E_{W}$.

We note that a Dirac structure on a real vector space $V$ induces a Dirac structure on every subspace of $V$ (see [Cou]). In our situation, however, not every subspace of a GC vector space is a GC subspace. The reason is the extra condition $E_{W} \cap \bar{E}_{W}=(0)$, which has no counterpart in the theory of Dirac structures.

\section{Examples 3.3.}

(1) Consider a complex GCS on $V$, corresponding to a complex structure $J$ on $V$. Then a real subspace $W \subseteq V$ is a GC subspace if and only if $W$ is stable under $J$. In this case, the induced GC structure on $W$ is also complex, corresponding to $\left.J\right|_{W}$.

(2) Consider a symplectic GCS on $V$, corresponding to a symplectic form $\omega$ on $V$. Then a real subspace $W \subseteq V$ is a GC subspace if and only if $\left.\omega\right|_{W}$ is nondegenerate on $W$. In this case, the induced GC structure on $W$ is also symplectic, corresponding to $\left.\omega\right|_{W}$.

This follows from Proposition 7.1, where we give a complete description of GC subspaces of $B$-complex and $B$-symplectic vector spaces.

Remark 3.4. In general, it seems that if $W$ is a GC subspace of a GC vector space $V$, then there is no simple relationship between $\mathcal{J}_{W}$ and $\mathcal{J}_{V}$. Later we will see that such a relationship exists for special types of GC subspaces. 
It is important to understand the passage from a GCS on $V$ to the induced GCS on a GC subspace $W \subseteq V$ in terms of spinors. To that end, we have the following

Proposition 3.5. Let $V$ be a real vector space with a $G C$ structure $E \subset$ $V_{\mathbb{C}} \oplus V_{\mathbb{C}}^{*}$. Let $W \subseteq V$ be any subspace, and let $j: W \hookrightarrow V$ denote the inclusion map. Then there exists a representative spinor for $E$ of the form $\phi=\exp (u) \wedge f_{1} \wedge \cdots \wedge f_{k}$, such that, for some $1 \leq l \leq k, j^{*}\left(f_{1}\right), \ldots, j^{*}\left(f_{l}\right)$ are a basis of $\operatorname{Ann}\left(\rho(E) \cap W_{\mathbb{C}}\right) \subseteq W_{\mathbb{C}}^{*}$, and $f_{l+1}, \ldots, f_{k}$ are a basis of $\operatorname{Ann}(\rho(E)+$ $\left.W_{\mathbb{C}}\right)$. Moreover, $\phi_{W}:=\exp \left(j^{*} u\right) \wedge j^{*}\left(f_{1}\right) \wedge \cdots \wedge j^{*}\left(f_{l}\right)$ is a representative spinor for $E_{W}$. In particular, $W$ is a $G C$ subspace of $V$ if and only if $\left\langle\phi_{W}, \bar{\phi}_{W}\right\rangle_{M} \neq 0$.

Corollary 3.6. Let $V$ be a real vector space, let $E$ be a $G C$ structure on $V$, and let $E^{\prime}$ be a $B$-field transform of $E$, defined by $B \in \wedge^{2} V^{*}$. Then a subspace $W \subseteq V$ is a GC subspace of $V$ with respect to $E$ if and only if it is a GC subspace with respect to $E^{\prime}$, and in that case, $E_{W}^{\prime}$ is a $B$-field transform of $E_{W}$ defined by $\left.B\right|_{W} \in \bigwedge^{2} W^{*}$.

Proof. Let $j: W \hookrightarrow V$ denote the inclusion map, and let $\phi=\exp (u) \wedge f_{1} \wedge$ $\cdots \wedge f_{k}$ be a pure spinor for the structure $E$, such that the $f_{i}$ 's satisfy the conditions of Proposition 3.5. It follows from Proposition 2.8 that the spinor $\phi^{\prime}=\exp (-B+u) \wedge f_{1} \wedge \cdots \wedge f_{k}$ is representative for $E^{\prime}$. Now Proposition 3.5 implies that

$$
\phi_{W}:=\exp \left(j^{*} u\right) \wedge j^{*}\left(f_{1}\right) \wedge \cdots \wedge j^{*}\left(f_{l}\right)
$$

and

$$
\phi_{W}^{\prime}:=\exp \left(-j^{*} B+j^{*} u\right) \wedge j^{*}\left(f_{1}\right) \wedge \cdots \wedge j^{*}\left(f_{l}\right)
$$

are representative spinors for $E_{W}$ and $E_{W}^{\prime}$, respectively. Since $B$ is real, it is immediate from Theorem 2.5(2.5) that $\left\langle\phi_{W}, \bar{\phi}_{W}\right\rangle_{M}=\left\langle\phi_{W}^{\prime}, \bar{\phi}^{\prime}{ }_{W}\right\rangle_{M}$, proving the first claim. The second assertion is clear.

Remark 3.7. This corollary could be obtained from a more general result of Bursztyn and Radko ([BR], Lemma 2.11). However, our proof differs from the one given in loc.cit.

3.2. GC quotients and duality. Let $V$ be a real vector space with a GCS defined by $E \subset V_{\mathbb{C}} \oplus V_{\mathbb{C}}^{*}$. Consider a (real) subspace $W \subseteq V$ and the corresponding quotient $V / W$. Let $\pi: V \rightarrow V / W$ be the projection map, and let $\eta: \operatorname{Ann}(W) \rightarrow(V / W)^{*}$ be the natural isomorphism. Define, dually to $(3.1)$,

$$
E_{V / W}=\left\{\left(\pi(\rho(e)), \eta\left(\rho^{*}(e)\right)\right) \mid e \in E \cap\left(V_{\mathbb{C}} \oplus \operatorname{Ann}\left(W_{\mathbb{C}}\right)\right)\right\} .
$$

Again, it is easy to check (cf. Lemma 8.2) that $E_{V / W}$ is a maximally isotropic subspace of $(V / W)_{\mathbb{C}} \oplus(V / W)_{\mathbb{C}}^{*}$. 
Definition 3.8. We say that $V / W$ is a generalized complex quotient of $V$ if $E_{V / W} \cap \bar{E}_{V / W}=(0)$. In this case, we denote by $\mathcal{J}_{V / W}$ the orthogonal automorphism of $(V / W) \oplus(V / W)^{*}$ corresponding to $E_{V / W}$.

Most questions about GC quotients can be easily reduced to questions about GC subspaces by means of the following result.

Proposition 3.9. Let $V$ be a real vector space with a fixed GCS given by $E \subset V_{\mathbb{C}} \oplus V_{\mathbb{C}}^{*}$. If $W \subseteq V$ is a real subspace, then $V / W$ is a $G C$ quotient of $V$ if and only if $\operatorname{Ann}(W)$ is a $G C$ subspace of $V^{*}$ with respect to $\tau(E)$ (cf. $\S 2.3)$. Suppose that this holds, and let $E_{V / W}$ be the induced $G C S$ on $V / W$. Let $E_{V / W}^{D}$ be the $G C S$ on $(V / W)^{*} \cong \operatorname{Ann}(W)$ induced by $\tau(E) \subset V_{\mathbb{C}}^{*} \oplus V_{\mathbb{C}}$, and let $\tau_{W}:(V / W) \oplus(V / W)^{*} \rightarrow(V / W)^{*} \oplus(V / W)$ be the isomorphism which interchanges the two summands. Then $E_{V / W}^{D}=\tau_{W}\left(E_{V / W}\right)$.

The proposition follows immediately from Lemma 8.1. One might expect at first that $W$ is a GC subspace of $V$ if and only if $V / W$ is a GC quotient of $V$. We have discovered that this is not so: see Example 7.6.

3.3. Classification of GC vector spaces. We begin by defining direct sums of $\mathrm{GC}$ vector spaces. Let $U, V$ be real vector spaces equipped with GC structures $E_{U} \subset U_{\mathbb{C}} \oplus U_{\mathbb{C}}^{*}, E_{V} \subset V_{\mathbb{C}} \oplus V_{\mathbb{C}}^{*}$. Let $\pi_{U}: U \oplus V \rightarrow U$, $\pi_{V}: U \oplus V \rightarrow V$ be the natural projections, and let

$$
\nu_{U, V}: U \oplus U^{*} \oplus V \oplus V^{*} \stackrel{\cong}{\longrightarrow}(U \oplus V) \oplus(U \oplus V)^{*}
$$

denote the obvious isomorphism (or its complexified version). The direct sum of the structures $E_{U}$ and $E_{V}$ is the GC structure $\nu_{U, V}\left(E_{U} \oplus E_{V}\right)$ on $U \oplus V$. If $E_{U}, E_{V}$ correspond to orthogonal automorphisms $\mathcal{J}_{U}, \mathcal{J}_{V}$, then the automorphism corresponding to the direct sum is $\nu_{U, V} \circ\left(\mathcal{J}_{U} \oplus \mathcal{J}_{V}\right) \circ \nu_{U, V}^{-1}$. Finally, if $\phi_{U}, \phi_{V}$ are representative spinors for $E_{U}$ and $E_{V}$, then $\pi_{U}^{*}\left(\phi_{U}\right) \wedge$ $\pi_{V}^{*}\left(\phi_{V}\right)$ is a representative spinor for the direct sum of the GC structures.

The main result of the subsection is the following "decomposition theorem."

Theorem 3.10. Every GCS is a B-field transform of a direct sum of a complex GCS and a symplectic GCS.

This decomposition is by no means unique. Let us describe the strategy of the proof of this theorem; along the way, we will give a more canonical (but weaker) version of the result. Suppose that $V$ is a GC vector space, with the GC structure defined by $E \subset V_{\mathbb{C}} \oplus V_{\mathbb{C}}^{*}$. Let $S$ be the subspace of $V$ such that $S_{\mathbb{C}}=\rho(E) \cap \rho(\bar{E})$. It is easy to check that $S$ is a GC subspace of $V$, and in fact, the maximal such subspace so that the induced structure on $S$ is $B$-symplectic. This subspace is the " $B$-symplectic part" of $V$, and is canonically determined. Moreover, $S$ doesn't change if we make a $B$-field 
transform of $V$. The next step of the proof is to choose an appropriate (nonunique) $B$-field transform of the whole structure on $V$ such that $S$ becomes "split", i.e., such that we can find a complementary GC subspace $W$ to $S$ in $V$ with $V=S \oplus W$ a direct sum of GC structures. To complete the proof, we show that $W$ is $B$-complex.

A dual construction is obtained by considering the subspace $C$ of $V$ such that $C_{\mathbb{C}}=\left(E \cap V_{\mathbb{C}}\right) \oplus\left(\bar{E} \cap V_{\mathbb{C}}\right)$. It may not be a GC subspace of $V$ (see Example 7.7); on the other hand, $V / C$ is always a GC quotient of $V$, and is $\beta$-symplectic. Note also that $C$ is obviously invariant under $\mathcal{J}$ (where $\mathcal{J}$ is the orthogonal automorphism of $V \oplus V^{*}$ corresponding to $E$ ), and $\left.\mathcal{J}\right|_{C}$ is a complex structure on $C$. Then we have the following result.

\section{Theorem 3.11.}

(a) Every $G C$ vector space $V$ has a unique maximal B-symplectic subspace $S$. If $V / S$ is a $G C$ quotient of $V$, then it is $B$-complex. In any case, $V / S$ can be endowed with a canonical complex structure.

(b) Dually, every $G C$ vector space $V$ has a unique minimal subspace $C$ such that $V / C$ is a $G C$ quotient which is $\beta$-symplectic. The subspace $C$ can be endowed with a canonical complex structure, and $C$ satisfies the graph condition with respect to this structure. If $C$ is a $G C$ subspace, the induced structure is $\beta$-complex.

Corollary 3.12. If $V$ is a $G C$ vector space, then $\operatorname{dim}_{\mathbb{R}} V$ is even.

Proof. By Theorem 3.11(b), there exists a subspace $C \subseteq V$ such that $C$ has a complex structure and $V / C$ is a $\beta$-symplectic GC quotient of $V$. Since a $\beta$ symplectic structure on a real vector space gives in particular a symplectic structure, it follows that $\operatorname{dim}_{\mathbb{R}} C$ and $\operatorname{dim}_{\mathbb{R}}(V / C)$ are even. Hence, so is $\operatorname{dim}_{\mathbb{R}} V$.

We end by giving an explicit formula for a general $B$-field transform of a direct sum of a symplectic and a complex GCS. Let $S$ be a real vector space with a symplectic form $\omega \in \bigwedge^{2} S^{*}$, let $C$ be a real vector space with a complex structure $J \in \operatorname{Aut}_{\mathbb{R}}(C)$, and form $V=S \oplus C$. We identify $V^{*}$ with $S^{*} \oplus C^{*}$ in the natural way. Let $B \in \bigwedge^{2} V^{*}$, which we view as a skew-symmetric map $B: V \rightarrow V^{*}$, and write accordingly as a matrix

$$
B=\left(\begin{array}{ll}
B_{1} & B_{2} \\
B_{3} & B_{4}
\end{array}\right)
$$

where $B_{1}: S \rightarrow S^{*}, B_{2}: C \rightarrow S^{*}, B_{3}: S \rightarrow C^{*}, B_{4}: C \rightarrow C^{*}$ are linear maps satisfying $B_{1}^{*}=-B_{1}, B_{4}^{*}=-B_{4}, B_{3}=-B_{2}^{*}$. In the result that follows, an automorphism $\mathcal{J}$ of $V \oplus V^{*}$ is viewed as a $4 \times 4$ matrix according to the decomposition $V \oplus V^{*}=S \oplus C \oplus S^{*} \oplus C^{*}$. 
Proposition 3.13. With this notation, let $\mathcal{J}$ be the automorphism of $V \oplus V^{*}$ corresponding to the GCS on $V$ which is the B-field transform of the direct sum of $(S, \omega)$ and $(C, J)$ defined by $B$. Then $\mathcal{J}$ is given by

$$
\mathcal{J}=\left(\begin{array}{cccc}
\omega^{-1} B_{1} & \omega^{-1} B_{2} & -\omega^{-1} & 0 \\
0 & J & 0 & 0 \\
\omega+B_{1} \omega^{-1} B_{1} & B_{2} J+B_{1} \omega^{-1} B_{2} & -B_{1} \omega^{-1} & 0 \\
B_{3} \omega^{-1} B_{1}+J^{*} B_{3} & B_{4} J+B_{3} \omega^{-1} B_{2}+J^{*} B_{4} & -B_{3} \omega^{-1} & -J^{*}
\end{array}\right) .
$$

If $\pi_{S}: V \rightarrow S, \pi_{C}: V \rightarrow C$ are the two projections, then the pure spinor corresponding to $\mathcal{J}$ is given by

$$
\phi=\exp \left(-B+i \pi_{S}^{*} \omega\right) \wedge \pi_{C}^{*}\left(f_{1}\right) \wedge \cdots \wedge \pi_{C}^{*}\left(f_{k}\right),
$$

where $f_{1}, \ldots, f_{k}$ are a basis of the $-i$-eigenspace of $J^{*}$ on $C_{\mathbb{C}}^{*}$.

The (entirely straightforward) proof is omitted. Observe that from this description, it follows that $\omega, J$ and $B_{1}, B_{2}, B_{3}$ can be recovered from $\mathcal{J}$, while $B_{4}$ can only be recovered up to the addition of a real bilinear skew form $\Omega$ on $C$ satisfying $\Omega J+J^{*} \Omega=0$.

\section{Split subspaces and the graph condition}

4.1. Opposite GC structures. Let $V$ be a real vector space, and consider a GC structure on $V$ defined in terms of an orthogonal automorphism $\mathcal{J}$ of $V \oplus V^{*}$ or the corresponding pure spinor $\phi \in \wedge^{\bullet} V_{\mathbb{C}}^{*}$. As in $\S 1.5$, we write $\mathcal{J}$ in matrix form:

$$
\mathcal{J}=\left(\begin{array}{cc}
\mathcal{J}_{1} & \mathcal{J}_{2} \\
\mathcal{J}_{3} & \mathcal{J}_{4}
\end{array}\right)
$$

We define the opposite of $\mathcal{J}$ to be the GC structure on $V$ defined by

$$
\tilde{\mathcal{J}}=\left(\begin{array}{cc}
\mathcal{J}_{1} & -\mathcal{J}_{2} \\
-\mathcal{J}_{3} & \mathcal{J}_{4}
\end{array}\right)
$$

It is easy to check that $\tilde{\mathcal{J}}^{2}=-1$ and that $\tilde{\mathcal{J}}$ is orthogonal with respect to the usual pairing $\langle\cdot, \cdot\rangle$ on $V \oplus V^{*}$. If $V$ and $W$ are GC vector spaces, with the GC structure given by $\mathcal{J}_{V}$ and $\mathcal{J}_{W}$, respectively, then by the twisted product structure on $W \oplus V$ we will mean the direct sum (cf. §3.3) of the GCS $\tilde{\mathcal{J}}_{W}$ on $W$ and the GCS $\mathcal{J}_{V}$ on $V$.

We can describe opposite GC structures in terms of spinors as follows.

Proposition 4.1. Suppose that the $G C$ structure $\mathcal{J}$ corresponds to the pure spinor $\phi$, written in standard form as $\phi=\exp (u) \wedge f_{1} \wedge \cdots \wedge f_{k}$ (cf. Theorem 2.5). Then $\tilde{\mathcal{J}}$ corresponds to the spinor $\tilde{\phi}=\exp (-u) \wedge f_{1} \wedge \cdots \wedge f_{k}$.

Proof. Using our classification of GC structures (Theorem 3.10), we may assume that $\mathcal{J}$ has the form (3.3). Then it it clear that replacing $\mathcal{J}$ with the opposite GC structure is the same as replacing $\omega, B_{1}, B_{2}, B_{3}$ and $B_{4}$ 
by their negatives. This operation replaces the spinor (3.4) by the spinor $\exp \left(B-i \pi_{S}^{*} \omega\right) \wedge \pi_{C}^{*}\left(f_{1}\right) \wedge \cdots \wedge \pi_{C}^{*}\left(f_{k}\right)$, whence the result.

Remark 4.2. It is easy to check (without going into the proof of Proposition 4.1) that the operation

$$
\phi=\exp (u) \wedge f_{1} \wedge \cdots \wedge f_{k} \mapsto \tilde{\phi}=\exp (-u) \wedge f_{1} \wedge \cdots \wedge f_{k}
$$

is well defined on pure spinors. Indeed, if

$$
\exp (u) \wedge f_{1} \wedge \cdots \wedge f_{k}=\exp (v) \wedge g_{1} \wedge \cdots \wedge g_{k},
$$

where $u, v$ are 2 -forms and $f_{i}, g_{j}$ are 1 -forms, then by equating the homogeneous parts of degree $k+2 m$ of both sides, we find that

$$
u^{m} \wedge f_{1} \wedge \cdots \wedge f_{k}=v^{m} \wedge g_{1} \wedge \cdots \wedge g_{k}
$$

for all $m \geq 0$, whence

$$
(-u)^{m} \wedge f_{1} \wedge \cdots \wedge f_{k}=(-v)^{m} \wedge g_{1} \wedge \cdots \wedge g_{k}
$$

for all $m \geq 0$, proving our observation.

\section{Examples 4.3.}

(1) The opposite of a complex GCS is the structure itself.

(2) The opposite of a symplectic GCS defined by a symplectic form $\omega$ is the symplectic GCS defined by $-\omega$.

(3) If $\mathcal{J}$ is a GCS on a real vector space $V$ and $\mathcal{J}^{\prime}$ is a $B$-field transform of $\mathcal{J}$ defined by $B \in \bigwedge^{2} V^{*}$, then $\tilde{\mathcal{J}}^{\prime}$ is the $B$-field transform of $\tilde{\mathcal{J}}$ defined by $-B$.

4.2. Generalized Lagrangian subspaces and the graph condition. We begin by defining three types of subspaces of GCS, the last of which was introduced (under the name "generalized complex subspace") by Gualtieri ([Gua $]$ ) and Hitchin $([\mathbf{H 3}])$.

Definition 4.4. Let $V$ be a real vector space with a GCS defined by $\mathcal{J} \in$ $\operatorname{Aut}_{\mathbb{R}}\left(V \oplus V^{*}\right)$, and let $W \subseteq V$ be a subspace.

(1) We call $W$ a generalized isotropic subspace if $\mathcal{J}(W) \subseteq W \oplus \operatorname{Ann}(W)$.

(2) We call $W$ a generalized coisotropic subspace if $\mathcal{J}(\operatorname{Ann}(W)) \subseteq W \oplus$ $\operatorname{Ann}(W)$.

(3) We call $W$ a generalized Lagrangian subspace if $W$ is both generalized isotropic and generalized coisotropic, that is, if $W \oplus \operatorname{Ann}(W)$ is stable under $\mathcal{J}$.

Examples 4.5. In the symplectic case, the three definitions specialize to usual isotropic, coisotropic and Lagrangian subspaces, respectively. In the complex case, all three definitions specialize to complex subspaces (i.e., the subspaces stable under the automorphism $J$ defining the complex structure). ${ }^{3}$

\footnotetext{
${ }^{3}$ For more details, see $\S 7.3$.
} 
Recall from classical symplectic geometry (see, e.g., $[\mathbf{C d S}]$ ) that if $\left(W, \omega_{W}\right)$ and $\left(V, \omega_{V}\right)$ are symplectic vector spaces, then a vector space isomorphism $W \rightarrow V$ is a symplectomorphism if and only if its graph is a Lagrangian subspace of $W \oplus V$ with respect to the "twisted product structure" on the direct sum, defined by the symplectic form $-\omega_{W}+\omega_{V}$. More generally, suppose $\left(V, \omega_{V}\right)$ is a symplectic vector space and $W \subseteq V$ is a subspace equipped with some symplectic form $\omega_{W}$. Then $\omega_{W}=\left.\omega_{V}\right|_{W}$ if and only if the graph of the inclusion $W \hookrightarrow V$ is an isotropic subspace of $W \oplus V$ with respect to the twisted product structure. This motivates the following

Definition 4.6. Let $V$ be a GC vector space, and let $W \subseteq V$ be a subspace equipped with a GC structure. We say that $W$ (together with the given GCS) satisfies the graph condition if the graph of the inclusion $W \hookrightarrow V$ is a generalized isotropic subspace of $W \oplus V$ with respect to the twisted product structure.

4.3. Split subspaces. Let $V$ be a real vector space with a GC structure defined by $\mathcal{J} \in \operatorname{Aut}_{\mathbb{R}}\left(V \oplus V^{*}\right)$. The following definition is somewhat similar in spirit to the definition of generalized Lagrangian subspaces.

Definition 4.7. We say that a subspace $W \subseteq V$ is split if there exists a subspace $N \subseteq V$ such that $V=W \oplus N$ and $W \oplus \operatorname{Ann}(N)$ is stable under $\mathcal{J}$.

The terminology is explained by the first part of the following result.

Proposition 4.8. Let $\mathcal{J}$ be a $G C S$ on a real vector space $V$, and let $W \subseteq V$ be a split subspace, so that $V=W \oplus N$ for some subspace $N \subseteq V$ such that $W \oplus \operatorname{Ann}(N)$ is stable under $\mathcal{J}$. Then

(a) Both $W$ and $N$ are GC subspaces of $V$. Moreover, $V=W \oplus N$ is a direct sum of $G C$ structures.

(b) Consider the natural isomorphism $\psi: W \oplus \operatorname{Ann}(N) \rightarrow W \oplus W^{*}$, given by $(w, f) \mapsto\left(w,\left.f\right|_{W}\right)$. Then the induced $G C S \mathcal{J}_{W}$ on $W$ has the form $\mathcal{J}_{W}=\psi \circ\left(\left.\mathcal{J}\right|_{W \oplus \operatorname{Ann}(N)}\right) \circ \psi^{-1}$.

(c) The space $N \oplus \operatorname{Ann}(W)$ is also stable under $\mathcal{J}$, and the induced structure $\mathcal{J}_{N}$ on $N$ has a similar description.

4.4. Relations between the various notions of subspaces. We have already seen six different notions of subspaces of GC vector spaces: GC subspaces, subspaces satisfying the graph condition, split subspaces, and generalized isotropic/coisotropic/Lagrangian subspaces. We will now describe in detail the relations between these types of subspaces. The significance of generalized Lagrangian subspaces as graphs of isomorphisms between GC vector spaces, and, more generally, the role of generalized isotropic subspaces as the key ingredient in our notion of the graph condition, has already been explained. The rest is contained in the following three results. 
Proposition 4.9. Suppose that $W$ is a split subspace of a $G C$ vector space $V$. Then $W$ satisfies the graph condition with the induced $G C$ structure.

Proposition 4.10. Suppose that $V$ is a GC vector space with the GCS defined by

$$
\mathcal{J}=\left(\begin{array}{cc}
\mathcal{J}_{1} & \mathcal{J}_{2} \\
\mathcal{J}_{3} & \mathcal{J}_{4}
\end{array}\right)
$$

Then a $G C$ subspace $W \subseteq V$ satisfies the graph condition (with respect to the induced structure) if and only if $W$ is stable under $\mathcal{J}_{1}$. Moreover, in this case, if $\mathcal{J}_{W}$ denotes the induced $G C$ structure on $W$, then

$$
\mathcal{J}_{W 1}=\left.\mathcal{J}_{1}\right|_{W} \text { and } \mathcal{J}_{W 3}(w)=\left.\mathcal{J}_{3}(w)\right|_{W} \forall w \in W .
$$

Proposition 4.11. Suppose that $\mathcal{J}$ is a $G C S$ on a real vector space $V$, and suppose that $W \subseteq V$ is a subspace equipped with a $G C S \mathcal{K}$. Then

(a) $W$ satisfies the graph condition with respect to $\mathcal{K}$ if and only if $\mathcal{K}_{1}=$ $\left.\mathcal{J}_{1}\right|_{W}\left(\right.$ in particular, $W$ is stable under $\mathcal{J}_{1}$ ) and $\mathcal{K}_{3}(w)=\left.\mathcal{J}_{3}(w)\right|_{W}$ for all $w \in W$.

Now suppose that $W$ does satisfy the graph condition with respect to $\mathcal{K}$, and assume that $W$ is also a GC subspace ${ }^{4}$ of $V$. Then

(b) if $\mathcal{J}_{W}$ denotes the induced $G C S$ on $W$, then $W$ also satisfies the graph condition with respect to $\mathcal{J}_{W}$; and

(c) $\mathcal{K}$ is a $\beta$-field transform of $\mathcal{J}_{W}$.

4.5. Final comments. Before moving on to the global counterpart of the theory developed above, we would like to mention several interesting examples and counterexamples that already appear on the vector space level. They are worked out in detail in Section 7.

- There exists a linear GCS which is both a $B$-field transform of a symplectic structure, and a $\beta$-field transform of a different symplectic structure.

- There exists a linear GCS which is both a $B$-field transform of a symplectic structure, and a $\beta$-field transform of a complex structure.

- In particular, there exist examples where one can start with a symplectic structure on a vector space and deform it continuously into a complex structure, through GC structures.

- There exists a $B$-symplectic vector space $V$ and a GC subspace $W \subset V$ such that $V / W$ is not a GC quotient of $V$. (This situation cannot occur if $V$ is either purely complex or purely symplectic.)

- There exists a $B$-symplectic vector space $V$ such that if $C$ is the minimal subspace of $V$ such that $V / C$ is a $\beta$-symplectic quotient of $V$ (cf. Theorem 3.11), then $C$ is not a GC subspace of $V$. However, this situation cannot occur if $V$ is $B$-complex.

\footnotetext{
${ }^{4}$ As we show in Remark 7.4, this condition is not automatic.
} 


\section{Generalized complex manifolds and submanifolds}

5.1. Summary. We begin the section by recalling the definitions of generalized almost complex and complex manifolds, following [Gua], [H2], [H3]. We slightly generalize the approach of Hitchin and Gualtieri by discussing generalized almost complex structures on vector bundles (other than the tangent bundle to a manifold). We then explain how integrability of a generalized complex structure on a manifold can be expressed in terms of the Courant bracket or the Courant-Nijenhuis tensor. We also mention that in

this more general approach, the right setting for defining integrability of generalized almost complex structures seems to be the one provided by the theory of Courant algebroids [LWX], [Roy].

We then study the analogue of Courant's notion of an admissible function [Cou], and we define the Courant sheaf, which we show to be a sheaf of local Poisson algebras.

Next we define the generalized complex submanifolds and split submanifolds, as well as generalized isotropic (resp. coisotropic, resp. Lagrangian) submanifolds of generalized complex manifolds. The theory is completely analogous to its linear counterpart, in that integrability here plays no role: the analogue of Courant's theorem, stating that the Dirac structure on a submanifold induced from an integrable Dirac structure on an ambient manifold is itself integrable, also holds in our situation.

We conclude the section by giving a justification for our notion of a split submanifold. Namely, we show that the sections of a Courant sheaf on a generalized complex manifold can be "pulled back" to a split submanifold, in a natural (though non-canonical) way. This was our original motivation for introducing the definition of a split submanifold.

5.2. Definitions of GAC manifolds. Let $M$ be a real manifold. Following $[\mathbf{G u a}]$ and $[\mathbf{H 1}]-[\mathbf{H 3}]$, we define a generalized almost complex structure (GACS) on a (finite rank) real vector bundle $V \rightarrow M$ to be one of the following two objects:

- A subbundle $E \subset V_{\mathbb{C}} \oplus V_{\mathbb{C}}^{*}$ which is maximally isotropic with respect to the standard pairing $\langle\cdot, \cdot\rangle$ and satisfies $E \cap \bar{E}=0$; or

- An $\mathbb{R}$-linear bundle automorphism $\mathcal{J}$ of $V \oplus V^{*}$ which is orthogonal with respect to $\langle\cdot, \cdot\rangle$ and satisfies $\mathcal{J}^{2}=-1$.

The equivalence of these two descriptions is proved in the same way as in the linear case (Proposition 2.1). If the tangent bundle $T M$ of $M$ is endowed with a GACS, we call $M$ a generalized almost complex manifold (GACM). It follows immediately from Corollary 3.12 that a GACM must have even dimension as a real manifold.

Remark 5.1. One can also describe GAC structures on vector bundles in terms of spinors. First observe that it is straightforward to generalize the 
theory described in $\S 2.2$ to the case of vector bundles on manifolds. Namely, if $V$ is a (finite rank) real vector bundle on a (real) manifold $M$ and $V_{\mathbb{C}}$ is its complexification, one naturally defines the "sheaf of Clifford algebras" Cliff $(V)$ which acts on the "bundle of spinors" $\wedge^{\bullet} V_{\mathbb{C}}^{*}$. If $E \subset V_{\mathbb{C}} \oplus V_{\mathbb{C}}^{*}$ is a maximally isotropic subbundle, it is easy to check (using Theorem 2.5(b)) that the subsheaf of $\wedge^{\bullet} V_{\mathbb{C}}^{*}$ consisting of the (germs of) sections that are annihilated by $E$ is a line subbundle of $\wedge^{\bullet} V_{\mathbb{C}}^{*}$. The Mukai pairing is also defined and becomes a pairing $\langle\cdot, \cdot\rangle_{M}: \bigwedge^{\bullet} V_{\mathbb{C}}^{*} \times \bigwedge^{\bullet} V_{\mathbb{C}}^{*} \rightarrow \bigwedge^{n} V_{\mathbb{C}}^{*}$, where $n$ is the rank of $V$. Thus, alternatively, a GACS on a real vector bundle $V \rightarrow M$ can be defined as the data of a line subbundle $\Phi \subseteq \bigwedge^{\bullet} V_{\mathbb{C}}^{*}$ such that, for every nonvanishing local section $\phi$ of $\Phi$, the function $\langle\phi, \bar{\phi}\rangle_{M}$ is nonvanishing, and $\phi$ is pointwise a pure spinor in the sense of Definition 2.4.

The following definition will be used in Section 6 .

Definition 5.2. If $V, W$ are real vector spaces equipped with GC structures $\mathcal{J}_{V} \in \operatorname{Aut}_{\mathbb{R}}\left(V \oplus V^{*}\right)$ and $\mathcal{J}_{W} \in \operatorname{Aut}_{\mathbb{R}}\left(W \oplus W^{*}\right)$, an isomorphism of $G C$ vector spaces between $V$ and $W$ if a linear isomorphism of real vector spaces $\varphi: V \stackrel{\sim}{\longrightarrow} W$ such that if $\varphi^{*}: W^{*} \stackrel{\sim}{\longrightarrow} V^{*}$ is the adjoint map, then

$$
\left(\varphi,\left(\varphi^{*}\right)^{-1}\right) \circ \mathcal{J}_{V}=\mathcal{J}_{W} \circ\left(\varphi,\left(\varphi^{*}\right)^{-1}\right),
$$

where $\left(\varphi,\left(\varphi^{*}\right)^{-1}\right)$ is naturally a map between $V \oplus V^{*}$ and $W \oplus W^{*}$.

If $M$ and $N$ are GAC manifolds, an isomorphism of GAC manifolds between $M$ and $N$ is a diffeomorphism $\phi: M \stackrel{\sim}{\longrightarrow} N$ such that for every $m \in M$, the differential $d \phi_{m}: T_{m} M \rightarrow T_{\phi(m)} N$ is an isomorphism between the induced linear GC structures on the tangent spaces.

5.3. Integrability. Recall that an almost complex structure on a manifold $M$ arises from an actual complex structure if a suitable integrability condition is satisfied (this is the Newlander-Nirenberg theorem). Hitchin defines "generalized complex structures" in terms of a natural generalization of that integrability condition. To formulate this condition, let us recall first the definition of the Courant bracket ([Cou], p. 645) on the sections of $T_{\mathbb{C}} M \oplus T_{\mathbb{C}}^{*} M$ :

$$
[X \oplus \xi, Y \oplus \eta]_{\mathrm{cou}}=[X, Y]+\mathcal{L}_{X} \eta-\mathcal{L}_{Y} \xi+\frac{1}{2} \cdot d\left(\iota_{Y} \xi-\iota_{X} \eta\right) .
$$

Here, $[X, Y]$ is the usual Lie bracket on vector fields.

Definition 5.3 (cf. [Gua], [H2], [H3]). Let $M$ be a real manifold equipped with a GACS defined by $E \subset T_{\mathbb{C}} M \oplus T_{\mathbb{C}}^{*} M$. We say that $E$ is integrable if the sheaf of sections of $E$ is closed under the Courant bracket. If that is the case, we also say that $E$ is a generalized complex structure on $M$, and that $M$ is a generalized complex manifold (GCM). 
Remark 5.4 (cf. [Cou], [H3]). The integrability condition for a GACS can be expressed in terms of the corresponding automorphism $\mathcal{J}$, in a way completely analogous to the usual almost complex case. Namely, integrability of a GACS defined by $\mathcal{J}$ is equivalent to the vanishing of the Courant-Nijenhuis tensor

$$
N_{\mathcal{J}}(X, Y)=[\mathcal{J} X, \mathcal{J} Y]_{\text {cou }}-\mathcal{J}[\mathcal{J} X, Y]_{\text {cou }}-\mathcal{J}[X, \mathcal{J} Y]_{\text {cou }}-[X, Y]_{\text {cou }}
$$

where $X, Y$ are sections of $T M \oplus T^{*} M$.

Integrability can also be expressed in terms of spinors; however, we will not use this description in our paper. We refer the reader to $[\mathbf{H 3}]$ for a discussion.

Examples 5.5 (cf. [Gua]).

1) Let $J$ be a usual almost complex structure on $M$. Applying pointwise the construction of Definition 2.10(a), we get a generalized almost complex structure on $M$. This structure is integrable in the sense of Definition 5.3 if and only if $J$ is integrable in the sense of the Newlander-Nirenberg theorem.

2) Let $\omega$ be a nondegenerate differential 2-form on $M$. Applying pointwise the construction of Definition 2.10(c), we get a generalized almost complex structure on $M$. This structure is integrable if and only if $\omega$ is closed, i.e., is a symplectic form on $M$.

We also note that there are global analogues of the notions of $B$ - and $\beta$-field transforms. For example, a closed 2 -form $B$ acts on GC structures on $M$ in the same way as described in $\S 2.4$. We refer the reader to [Gua] for more details. Our terminology in the global case will be the same as in the linear case; thus, for instance, we will refer to a $B$-field transform of a complex (resp., symplectic) manifold as a $B$-complex (resp., B-symplectic) GCM.

Remark 5.6. Recall that in symplectic geometry, it is sometimes important to consider symplectic forms on vector bundles over a manifold, other than its tangent bundle (see $[\mathbf{V}]$ ). Thus, if $V$ is a (finite rank) real vector bundle on a real manifold $M$, one says that $V$ is a symplectic vector bundle if $V$ is equipped with a nondegenerate 2 -form $\omega \in \Gamma\left(M, \bigwedge^{2} V^{*}\right)$. One can then ask for a generalization of the closedness condition on $\omega$. An appropriate setting for this is provided by the notion of a Lie algebroid (cf., e.g., [Roy]). A Lie algebroid structure on the vector bundle $V$ gives a degree 1 differential $d_{V}$ on the sheaf of sections of $\Lambda^{\bullet} V^{*}$, making the latter a sheaf of differential graded algebras. The definition of $d_{V}$ is given by the usual Leibniz formula (cf. $[\mathbf{R o y}]$ or $[\mathbf{N T}]$ ). One can now define a symplectic Lie algebroid (cf. [NT]) to be a Lie algebroid $V$, together with a nondegenerate 2-form $\omega \in$ $\Gamma\left(M, \bigwedge^{2} V^{*}\right)$, such that $d_{V} \omega=0$. By taking $V=T M$ with its standard Lie algebroid structure, we recover the usual notion of a symplectic manifold. 
In view of these remarks, it seems that an appropriate general setting for the study of generalized complex structures would be the one provided by the theory of Courant algebroids (we refer the reader to [Roy] for a detailed study of these objects). We will not give the definition of a Courant algebroid, as it is rather long and is not used anywhere in our paper. Let us only remark that, if $V$ and $V^{*}$ are a pair of Lie algebroids in duality ([LWX $]$ or $[\mathbf{R o y}])$, then the direct sum $V \oplus V^{*}$ acquires a natural structure of a Courant algebroid $([\mathbf{L W X}])$, and part of this structure is a bracket $[\cdot, \cdot]$ on the sections of $V \oplus V^{*}$ which specializes to the Courant bracket in case $V=T M$ (with the usual Lie bracket, and with the anchor being the identity map), $V^{*}=T^{*} M$ (with zero bracket and anchor). Then one can define a GACS $E \subset V_{\mathbb{C}} \oplus V_{\mathbb{C}}^{*}$ to be integrable if the sheaf of sections of $E$ is closed under this bracket. In the case where $V=T M$, one recovers Definition 5.3.

5.4. The Courant sheaf. Let $M$ be a GACM, with the GACS defined by a maximally isotropic subbundle $E \subset T_{\mathbb{C}} M \oplus T_{\mathbb{C}}^{*} M$. We define the Courant sheaf of $M$ to be the following subsheaf $\mathcal{C O U}_{M}$ of $C_{M}^{\infty} \oplus T_{\mathbb{C}} M$ :

$$
\mathcal{C O U}_{M}(U)=\left\{(f, X) \in C_{M}^{\infty}(U) \oplus \Gamma\left(U, T_{\mathbb{C}} M\right) \mid d f+X \in \Gamma(U, \bar{E})\right\},
$$

for every open subset $U \subseteq M$. The origin of this definition lies in Courant's notion ([Cou $]$ ) of an admissible function. The precise analogue of this notion in our situation is the following: if $U \subseteq M$ is open, a function $f \in C_{M}^{\infty}(U)$ is admissible if there exists an $X \in \Gamma\left(U, T_{\mathbb{C}} M\right)$ with $(f, X) \in \mathcal{C O} \mathcal{U}_{M}(U)$.

The main properties of the Courant sheaf are summarized in the following

Proposition 5.7. Let $M$ be a generalized almost complex manifold.

(a) The Courant sheaf is closed under componentwise addition, and under the multiplication defined by

$$
(f, X) \cdot(g, Y)=(f \cdot g, f \cdot Y+g \cdot X) .
$$

Thus, $\mathcal{C O U}_{M}$ is a sheaf of (commutative unital) $\mathbb{C}$-algebras. In particular, the product of two admissible functions is admissible.

(b) Furthermore, $\mathrm{COU}_{M}$ is a sheaf of local rings. Namely, if $m \in M$, then the maximal ideal of the stalk $\mathcal{C O U}_{M, m}$ is formed by the germs of all sections $(f, X)$ of $\mathcal{C O U}_{M, m}$, defined near $m$, such that $f(m)=0$.

(c) Assume that $M$ is a generalized complex manifold. Then the Courant sheaf is also closed under the bracket defined by

$$
\{(f, X),(g, Y)\}=\left(\frac{1}{2} \cdot(X(g)-Y(f)),[X, Y]\right)=(X(g),[X, Y])
$$

(the last equality uses the fact that $\bar{E}$ is isotropic with respect to the standard pairing $\langle\cdot, \cdot\rangle)$, where $[\cdot, \cdot]$ is the usual Lie bracket of vector fields. With this operation, $\mathcal{C O U}_{M}$ is a sheaf of Poisson algebras. 
Part (c) of the proposition can be used to define a Poisson bracket on the sheaf of admissible functions, as was done in [Cou]. In $\S 5.6$, we will see that sections of the Courant sheaf can be pulled back to a split submanifold.

Remark 5.8. All the results of Proposition 5.7 are valid for arbitrary complex Dirac structures; in fact, as the reader can easily verify, our proof of the proposition does not use the "non-degeneracy condition" $E \cap \bar{E}=0$.

5.5. Submanifolds. Let $S$ be a smooth submanifold of a real manifold $M$, and let $E \subset T_{\mathbb{C}} M \oplus T_{\mathbb{C}}^{*} M$ be a GACS. Applying pointwise the construction of $\S 3.1$ to the vector bundle $\left.T M\right|_{S}$ with the GACS $\left.E\right|_{S}$ and the subbundle $\left.T S \subseteq T M\right|_{S}$, we obtain a maximally isotropic distribution $E_{S} \subset T_{\mathbb{C}} S \oplus T_{\mathbb{C}}^{*} S$. That is, $E_{S}$ is a subset of $T_{\mathbb{C}} S \oplus T_{\mathbb{C}}^{*} S$ such that the fiber of $E_{S}$ over every point $s \in S$ is a maximally isotropic subspace of $T_{s, \mathbb{C}} M \oplus T_{s, \mathbb{C}}^{*} M$.

Caution! In general, $E_{S}$ is not a subbundle of $T_{\mathbb{C}} S \oplus T_{\mathbb{C}}^{*} S$.

We refer the reader to [Cou] for a detailed discussion of the analogous situation for submanifolds of Dirac manifolds. In particular, Courant's arguments can be easily adapted to give a necessary condition under which $E_{S}$ is a subbundle of $T_{\mathbb{C}} S \oplus T_{\mathbb{C}}^{*} S$ (cf. [Cou], Theorem 3.1.1), and to prove that if $E_{S}$ is a subbundle and $E$ is integrable, then so is $E_{S}$ (cf. [Cou], Corollary 3.1.4). This fact will not be used in the sequel.

Definition 5.9. We say that $S$ is a generalized almost complex submanifold of $M$ if $E_{S}$ is a subbundle of $T_{\mathbb{C}} S \oplus T_{\mathbb{C}}^{*} S$ such that $E_{S} \cap \bar{E}_{S}=0$. If moreover $M$ is a GCM, we will simply say that $S$ is a generalized complex submanifold of $M$.

We note, once again, that we have used different terminology than the one introduced by Gualtieri and Hitchin in [Gua], [H3]. They call a "generalized complex submanifold" what we call a "generalized Lagrangian submanifold" in the definition below.

Definition 5.10. Let $M$ be a generalized (almost) complex manifold, with the generalized (almost) complex structure defined by an orthogonal automorphism $\mathcal{J}$ of $T M \oplus T^{*} M$. Let $S$ be a smooth submanifold of $M$, and let Ann $(T S)$ be the annihilator of the subbundle $\left.T S \subseteq T M\right|_{S}$ in $\left.T^{*} M\right|_{S}$.

(1) We say that $S$ is a generalized isotropic submanifold of $M$ if $\mathcal{J}(T S) \subseteq$ $T S \oplus \operatorname{Ann}(T S)$.

(2) We say that $S$ is a generalized coisotropic submanifold of $M$ if $\mathcal{J}(\operatorname{Ann}(T S)) \subseteq T S \oplus \operatorname{Ann}(T S)$.

(3) We say that $S$ is a generalized Lagrangian submanifold of $M$ if $S$ is both generalized isotropic and generalized coisotropic, i.e., if $T S \oplus \operatorname{Ann}(T S)$ is stable under $\mathcal{J}$. 
5.6. Split submanifolds. Let $M$ be a generalized (almost) complex manifold, with the generalized (almost) complex structure defined by an orthogonal automorphism $\mathcal{J}$ of $T M \oplus T^{*} M$. We now define the global analogue of the notion of a split subspace introduced in $\S 4.3$.

Definition 5.11. A smooth submanifold $S$ of $M$ is said to be split if there exists a smooth subbundle $N$ of $\left.T M\right|_{S}$ such that $\left.T M\right|_{S}=T S \oplus N$, and $T S \oplus \operatorname{Ann}(N)$ is invariant under $\mathcal{J}$.

The following result is the global version of, and immediately follows from, Proposition 4.8 .

Proposition 5.12. Let $\mathcal{J} \in \operatorname{Aut}_{\mathbb{R}}\left(T M \oplus T^{*} M\right)$ be a GACS on a real manifold $M$, and let $S \subset M$ be a split submanifold, so that $\left.T M\right|_{S}=T S \oplus N$ for some subbundle $\left.N \subseteq T M\right|_{S}$ such that $T S \oplus \operatorname{Ann}(N)$ is stable under $\mathcal{J}$. Then $S$ is a GAC submanifold of $M$. Moreover, if $\psi: T S \oplus \operatorname{Ann}(N) \rightarrow T S \oplus T^{*} S$ is the natural isomorphism, then the induced GACS $\mathcal{J}_{S}$ on $S$ has the form $\mathcal{J}_{S}=\psi \circ\left(\left.\mathcal{J}\right|_{T S \oplus \operatorname{Ann}(N)}\right) \circ \psi^{-1}$.

The last result of the section relates split submanifolds to the Courant sheaf:

Theorem 5.13. Let $M, S$ and $\left.N \subseteq T M\right|_{S}$ be as above. Let $\pi:\left.T_{\mathbb{C}} M\right|_{S} \rightarrow$ $T_{\mathbb{C}} S$ denote the projection parallel to $N_{\mathbb{C}}$. For every open subset $U \subseteq M$, the map

$$
\Gamma\left(U, C_{M}^{\infty} \oplus T_{\mathbb{C}} M\right) \longrightarrow \Gamma\left(U \cap S, C_{S}^{\infty} \oplus T_{\mathbb{C}} S\right),
$$

given by $(f, X) \mapsto\left(\left.f\right|_{S}, \pi\left(\left.X\right|_{S}\right)\right)$, induces a ring homomorphism from $\mathcal{C O U}_{M}(U)$ to $\mathcal{C O U}_{S}(U \cap S)$. In particular, the restriction of an admissible function to a split submanifold is always admissible.

\section{The generalized complex "category"}

6.1. The graph condition. We begin by defining twisted product structures for manifolds. Let $M, N$ be real manifolds equipped with GAC structures defined by $\mathcal{J}_{M} \in \operatorname{Aut}_{\mathbb{R}}\left(T M \oplus T^{*} M\right), \mathcal{J}_{N} \in \operatorname{Aut}_{\mathbb{R}}\left(T N \oplus T^{*} N\right)$. Applying pointwise the construction of $\S 4.1$, we obtain the notion of the opposite structure of $\mathcal{J}_{M}$, which we will denote by $\tilde{\mathcal{J}}_{M}$. It is easy to check that the opposite of an integrable GACS is again integrable. We then define the twisted product structure on $M \times N$ to be the product of the GAC structures defined by $\tilde{\mathcal{J}}_{M}$ and $\mathcal{J}_{N}$. (We leave to the reader to define the notion of a product of GAC structures, which is, again, completely analogous to the notion of a direct sum of GC structures on vector spaces, defined in $§ 3.3$. Note also that the product of two integrable GAC structures is integrable.)

Now let $M$ be a GAC manifold, and let $j: S \hookrightarrow M$ be a smooth submanifold. Suppose that $S$ is equipped with some GACS. We then say that 
$S$, together with this GACS, satisfies the graph condition, if the graph of $j$ is a generalized isotropic submanifold of $S \times M$ with respect to the twisted product structure. We leave it to the reader to formulate the suitable global analogues of the results of $\S 4.4$. Let us only remark that this definition must be used with some caution (cf. also $\S 1.2$ in the introduction). For instance, if $j: S \rightarrow M$ is a diffeomorphism between two real manifolds, and both $S$ and $M$ are equipped with GAC structures such that $j$ satisfies the graph condition, then it does not follow that $j$ induces an isomorphism between the two GAC structures. The reason is that, in general (unlike the symplectic case) it is not true that a generalized isotropic submanifold, which has half the dimension of the ambient manifold, is generalized Lagrangian. However, we have the following result, which motivates the definition of the next subsection.

Theorem 6.1. Let $\phi: M \rightarrow N$ be a diffeomorphism of real manifolds, and suppose that $M, N$ are equipped with GAC structures. If the graph of $\phi$ is generalized Lagrangian with respect to the twisted product structure on $M \times N$, then $\phi$ induces an isomorphism between the two GAC structures.

Remark 6.2. Using the fact that $T(\operatorname{Graph}(\phi))$ is the image of the map $\left(1, \phi_{*}\right)$ from $T M$ to $T(M \times N)$ and that $\operatorname{Ann}(T(\operatorname{Graph}(\phi))$ is the image of the map $\left(-\phi^{*}, 1\right)$ from $T^{*}(N)$ to $T^{*}(M \times N)$, it is easily seen that, with respect to the twisted product structure on $M \times N$, the graph of $\phi$ is generalized isotropic if and only if

$$
\phi_{*} \mathcal{J}_{M 1}=\mathcal{J}_{N 1} \phi_{*} \text { and } \phi^{*} \mathcal{J}_{N 3} \phi_{*}=\mathcal{J}_{M 3}
$$

generalized coisotropic if and only if

$$
\phi_{*} \mathcal{J}_{M 2} \phi^{*}=\mathcal{J}_{N 2} \text { and } \phi^{*} \mathcal{J}_{N 4}=\mathcal{J}_{M 4} \phi^{*}
$$

and therefore generalized Lagrangian if and only if all of these equations hold.

6.2. Definition of the GC "category". We now introduce a "category" that generalizes Weinstein's definition [W2] of the symplectic "category." Suppose that $M, N$ are generalized complex manifolds. We define a canonical relation from $M$ to $N$ to be a generalized Lagrangian submanifold of $M \times N$ with respect to the twisted product structure. Now suppose that $P$ is a third GCM, and let $\Gamma \subset M \times N, \Phi \subset N \times P$ be canonical relations. The set-theoretic composition of $\Phi$ and $\Gamma$ is the subset

$$
\Phi \circ \Gamma=\{(m, p) \in M \times P \mid \exists n \in N \text { with }(m, n) \in \Gamma \text { and }(n, p) \in \Phi\} .
$$

If $\Phi \circ \Gamma$ is a generalized Lagrangian submanifold of $M \times P$ with respect to the twisted product structure, we say that $\Phi$ and $\Gamma$ are composable. This condition can be expressed in terms of a certain transversality property. We then define the generalized complex "category" to be the category whose 
objects are GC manifolds, and whose morphisms are the canonical relations. The composition is only defined on pairs of composable relations; thus, what we get is not a "true" category.

The details of this construction will be discussed in our subsequent publications.

6.3. The category of GC vector spaces. We finish the theoretical part of our paper by discussing the linear counterpart of the category introduced in $\S 6.2$. Our exposition here follows [GS]. If $V, W$ are real vector spaces, a relation from $V$ to $W$ is a linear subspace of $V \oplus W$. Let $Z$ be a third real vector space, and let $\Gamma \subset V \oplus W, \Phi \subset W \oplus Z$ be relations. The composition of $\Phi$ and $\Gamma$ is defined as is $\S 6.2$ (see also [GS], p. 942):

$$
\Phi \circ \Gamma=\{(v, z) \in V \oplus Z \mid \exists w \in W \text { with }(v, w) \in \Gamma \text { and }(w, z) \in \Phi\} .
$$

It is clear that $\Phi \circ \Gamma$ is a relation from $V$ to $Z$. The main result of the subsection is the following

Theorem 6.3. Let $V, W, Z$ be generalized complex vector spaces, and let $\Gamma \subset V \oplus W, \Phi \subset W \oplus Z$ be relations.

(a) If $\Gamma, \Phi$ are generalized isotropic subspaces with respect to the twisted product structures on $V \oplus W$ and $W \oplus Z$, then $\Phi \circ \Gamma$ is a generalized isotropic subspace with respect to the twisted product structure on $V \oplus$ $Z$.

(b) Same statement as part (a), with "generalized isotropic" replaced by "generalized coisotropic."

(c) Same statement as part (a), with "generalized isotropic" replaced by "generalized Lagrangian."

This theorem allows us to introduce the following

Definition 6.4 (The category $\underline{\text { Can }}$ ).

(1) If $V, W$ are GC vector spaces, a canonical relation from $V$ to $W$ is a generalized Lagrangian subspace of $V \oplus W$ with respect to the twisted product structure.

(2) The category Can of generalized complex vector spaces is defined as follows. The objects of Can are generalized complex vector spaces. If $V, W$ are objects of $\underline{\mathrm{Can}}$, the set of morphisms from $V$ to $W$ is the set of canonical relations from $V$ to $W$. The composition of morphisms is defined by (6.1).

\section{Examples and counterexamples}

7.1. Preliminaries. In this section we illustrate the main constructions and results of the previous sections with examples (and counterexamples). Following the philosophy of [Gua], we consider two basic types of examples: 
$B$-complex and $B$-symplectic vector spaces. In particular, as a result of our study, we will see that the three types of subspaces we have introduced (GC subspaces, GC subspaces satisfying the graph condition with respect to the induced structure, and split subspaces) are in general all distinct.

We begin by recalling explicit formulas for $B$ - and $\beta$-field transforms of symplectic structures. We refer to [Gua] for the (straightforward) computations. Let $V$ be a real vector space and let $B \in \bigwedge^{2} V^{*}, \beta \in \bigwedge^{2} V$. If $J$ is a complex structure on $V$ and $\mathcal{J}_{J}$ is the corresponding GCS (see Definition 2.10(a)), then the transforms of $\mathcal{J}$ defined by $B$ and $\beta$ are given by

$$
\left(\begin{array}{cc}
J & 0 \\
B J+J^{*} B & -J^{*}
\end{array}\right) \text { and }\left(\begin{array}{cc}
J & -J \beta-\beta J^{*} \\
0 & -J^{*}
\end{array}\right),
$$

respectively. If $\omega$ is a symplectic form on $V$ and $\mathcal{J}_{\omega}$ is the corresponding GCS (see Definition 2.10(c)), then the transforms of $\mathcal{J}$ defined by $B$ and $\beta$ are given by

$$
\left(\begin{array}{cc}
\omega^{-1} B & -\omega^{-1} \\
\omega+B \omega^{-1} B & -B \omega^{-1}
\end{array}\right) \quad \text { and } \quad\left(\begin{array}{cc}
\beta \omega & -\beta \omega \beta-\omega^{-1} \\
\omega & -\omega \beta
\end{array}\right)
$$

respectively.

It is also easy to describe $B$-field transforms of complex and symplectic structures in terms of the subspace $E \subset V_{\mathbb{C}} \oplus V_{\mathbb{C}}^{*}$. Let us start with the complex case. If $J$ is a complex structure on $V$, we call the elements of the $+i$-eigenspace (resp., $-i$-eigenspace) of $J$ in $V_{\mathbb{C}}$ the holomorphic (resp., antiholomorphic) vectors; and we call the elements of the $+i$-eigenspace (resp., - $i$-eigenspace) of $J^{*}$ in $V_{\mathbb{C}}^{*}$ the holomorphic (resp., antiholomorphic) 1 -forms on $V_{\mathbb{C}}$. Then the $B$-field transform of the complex GCS defined by $J$ is given by

$$
E=\left\{\left(v, \iota_{v} B+f\right) \mid v \in V_{\mathbb{C}} \text { is holomorphic, } f \in V_{\mathbb{C}}^{*} \text { is antiholomorphic }\right\} .
$$

On the other hand, if $\omega$ is a symplectic form on $V$, then the $B$-field transform of the symplectic GCS defined by $\omega$ is given by

$$
E=\left\{\left(v, \iota_{v}(B-i \omega)\right) \mid v \in V_{\mathbb{C}}\right\} .
$$

7.2. Subspaces, quotients and submanifolds. Let us classify GC subspaces of $B$-complex and $B$-symplectic vector spaces.

Proposition 7.1. Let $V$ be a real vector space, let $B \in \bigwedge^{2} V^{*}$, and let $W \subseteq V$ be a subspace.

(a) If $J$ is a complex structure on $V$ and $\mathcal{J}_{J}$ is the associated GCS, then $W$ is a $G C$ subspace of $V$ with respect to the transform of $\mathcal{J}_{J}$ defined by $B$ if and only if $W$ is invariant under $J$. In this case, the induced structure on $W$ is also B-complex, namely, it is the transform by $\left.B\right|_{W}$ of the complex GCS associated to $\left.J\right|_{W}$. The induced structure satisfies the graph condition. Furthermore, $W$ is split if and only if there exists 
a complement $N$ to $W$ in $V$ such that $N$ is also invariant under $J$, and $N$ is orthogonal to $W$ with respect to $B$.

(b) If $W$ is a subspace of a $B$-complex $G C$ vector space which satisfies the graph condition with respect to some $G C$ structure, then $W$ is also a GC subspace.

(c) If $\omega$ is a symplectic form on $V$ and $\mathcal{J}_{\omega}$ is the associated GCS, then $W$ is a GC subspace of $V$ with respect to the transform of $\mathcal{J}_{\omega}$ defined by $B$ if and only if $\left.\omega\right|_{W}$ is nondegenerate. In this case, the induced structure on $W$ is also B-symplectic, namely, it is the transform by $\left.B\right|_{W}$ of the symplectic GCS associated to $\left.\omega\right|_{W}$. Furthermore, $W$ is split if and only if the induced structure satisfies the graph condition, and either of these is equivalent to: if $N$ is the orthogonal complement to $W$ in $V$ with respect to $\omega$, then $N$ is also orthogonal to $W$ with respect to $B$.

The analogue of part (b) holds in the symplectic case, but fails in the more general $B$-symplectic case (cf. Remark 7.4). Using duality, it is easy to obtain a similar description of quotients of $\beta$-complex and $\beta$-symplectic structures. We omit the simple proof of Proposition 7.1, which is a combination of the results of Propositions 4.9-4.11 and Corollary 3.6, and the explicit formulas of $\S 7.1$.

This classification of GC subspaces generalizes without difficulty to a classification of GC submanifolds of $B$-complex and $B$-symplectic manifolds. Thus, for example, if $M$ is endowed with a $B$-field transform of a complex structure $J \in \operatorname{Aut}_{\mathbb{R}}(T M)$, then a submanifold $j: S \hookrightarrow M$ is generalized complex if and only if $\left.T S \subseteq T M\right|_{S}$ is stable under $J$. It is easy to check that the condition that $E_{S}$ be a smooth subbundle of $T_{\mathbb{C}} S \oplus T_{\mathbb{C}}^{*} S$ (cf. $\S 5.5$ ) is automatic. The induced structure on $S$ is also $B$-complex, defined by $\left.J\right|_{T S}$ and $j^{*} B$. A similar remark applies in the $B$-symplectic case. We leave to the reader the task of formulating the suitable descriptions of submanifolds satisfying the graph condition, and of split submanifolds, completely analogous to Proposition 7.1.

Finally, we observe that all GC submanifolds of complex or symplectic GC manifolds are already split. In the symplectic case, the "splitting" is canonical. More precisely, if $S$ is a GC submanifold of a symplectic manifold $(M, \omega)$, then there exists a unique subbundle $\left.N \subset T M\right|_{S}$ such that $\left.T M\right|_{S}=$ $T S \oplus N$ is a direct sum of GC structures; namely, $N$ is the orthogonal complement to $T S$ with respect to $\omega$. In the complex case, the "splitting" may not be unique, but there always exists one. Indeed, let $J$ be a complex structure on a real manifold $M$, and let $S$ be a submanifold of $M$ such that $\left.T S \subseteq T M\right|_{S}$ is $J$-invariant. Then $J$ also acts on the normal bundle 
$\mathcal{N}=\left(\left.T M\right|_{S}\right) / T S$, so we get a short exact sequence

$$
\left.0 \longrightarrow T S \longrightarrow T M\right|_{S} \longrightarrow \mathcal{N} \longrightarrow 0 .
$$

Since we are working in the $C^{\infty}$ category, there is a smooth splitting of this sequence $\sigma:\left.\mathcal{N} \rightarrow T M\right|_{S}$. We only need to make $\sigma$ equivariant with respect to $J$; since $J^{4}=1$, this is easily achieved by replacing $\sigma$ with (1/4) . $\sum_{k=0}^{3} J^{k} \circ \sigma \circ J^{-k}$.

7.3. Generalized Lagrangian subspaces and submanifolds. We now briefly discuss generalized isotropic, coisotropic, and Lagrangian subspaces (resp., submanifolds) of $B$-complex and $B$-symplectic GC vector spaces (resp., GC manifolds). First let $J$ be a complex structure on a real vector space $V$, inducing a complex GCS as usual. From Definition 2.10(a), it is clear that a subspace $W \subseteq V$ is generalized isotropic if and only if $J(W) \subseteq$ $W$, and it is generalized coisotropic if and only if $J^{*}(\operatorname{Ann}(W)) \subseteq \operatorname{Ann}(W)$. The latter condition is again equivalent to $J(W) \subseteq W$. Thus, in the complex case, the following classes of subspaces are all the same: generalized isotropic, generalized coisotropic, generalized Lagrangian, and generalized complex; and they all coincide with the class of subspaces invariant under $J$.

Next let $\omega$ be a symplectic form on $V$, inducing a symplectic GCS as usual. From Definition 2.10(c), it is clear that a subspace $W \subseteq V$ is generalized isotropic if and only if $\omega(W) \subseteq \operatorname{Ann}(W)$, i.e., $\left.\omega\right|_{W} \equiv 0$, that is, $W$ is isotropic in the sense of classical symplectic geometry. Similarly, $W$ is generalized coisotropic if and only if $W$ is coisotropic in the classical sense; and hence $W$ is generalized Lagrangian if and only if $W$ is Lagrangian.

These remarks carry over to the global case without any changes. Thus, if $M$ is a complex GCM, then the generalized isotropic (resp., coisotropic or Lagrangian) submanifolds of $M$ are precisely the complex submanifolds in the usual sense. Similarly, if $M$ is a symplectic GCM, then the generalized isotropic (resp. coisotropic, resp. Lagrangian) submanifolds of $M$ are the submanifolds that are isotropic (resp. coisotropic, resp. Lagrangian) in the classical sense.

Finally, we consider the case of $B$-complex and $B$-symplectic GC manifolds. For simplicity, we restrict ourselves to generalized Lagrangian submanifolds, which are studied in detail (under the name of "generalized complex submanifolds") in [Gua]. Thus, we only list the results, following [Gua].

- If $M$ is endowed with a GCS which is a transform of a complex structure on $M$ by a $B$-field $B \in \Gamma\left(M, \bigwedge^{2} T^{*} M\right)$, then a submanifold $j: S \hookrightarrow M$ is a generalized Lagrangian submanifold if and only if $S$ is a complex submanifold of $M$, and $j^{*} B$ is a form of type $(1,1)$ on $S$. 
- If $M$ is endowed with a GCS which is a transform of a symplectic structure on $M$ by a $B$-field $B \in \Gamma\left(M, \bigwedge^{2} T^{*} M\right)$, then a submanifold $j: S \hookrightarrow M$ is a generalized Lagrangian submanifold if and only if $S$ is a coisotropic submanifold of $M$ (in the classical sense), and, if $T S^{\perp}$ denotes the orthogonal complement to $T S$ in $\left.T M\right|_{S}$ with respect to the symplectic form on $M$, then $T S$ is orthogonal to $T S^{\perp}$ with respect to $B$, so that $j^{*} B$ descends to $T S / T S^{\perp}$; and, finally, $\left(j^{*} \omega\right)^{-1} j^{*} B$ is an almost complex structure on $T S / T S^{\perp}$.

7.4. Symplectic geometry with $B$-fields. Let $V$ be a real vector space, and let $\omega$ be a symplectic form on $V$. We have an injective linear map

$$
\bigwedge^{2} V^{*} \longrightarrow \operatorname{End}_{\mathbb{R}}(V)
$$

given by $B \mapsto T:=\omega^{-1} B$. A necessary and sufficient condition for $T \in$ $\operatorname{End}_{\mathbb{R}}(V)$ to be in the image of this map is that $(\omega T)^{*}=-\omega T$, or, equivalently,

$$
\omega(u, T v)=\omega(T u, v) \quad \forall u, v \in V .
$$

If $T$ satisfies (7.1), so that $T=\omega^{-1} B$ for some $B \in \bigwedge^{2} V^{*}$, then the $B$-field transform of the symplectic GCS defined by $\omega$ can be written in terms of $T$ as follows:

$$
\left(\begin{array}{cc}
T & -\omega^{-1} \\
\omega \circ\left(1+T^{2}\right) & -T^{*}
\end{array}\right)
$$

Thus, from Proposition 2.11, we immediately get the following

Proposition 7.2. The B-field transform of $\omega$ is

- symplectic $\Longleftrightarrow T=0$;

- $\beta$-symplectic $\Longleftrightarrow i$ is not an eigenvalue of $T$;

- $\beta$-complex $\Longleftrightarrow T^{2}=-1$.

The second possibility can be realized, e.g., by taking $T=1$ (which corresponds to $B=\omega$ ). This shows that a (nontrivial) $B$-field transform of a symplectic GCS may end up being $\beta$-symplectic as well. To show that the third possibility can also be realized, let us choose a basis $\left\{e_{1}, \ldots, e_{n}, f_{1}, \ldots, f_{n}\right\}$ of $V$ in which $\omega$ has the canonical form:

$$
\omega\left(e_{i}, f_{j}\right)=\delta_{i j}, \quad \omega\left(e_{i}, e_{j}\right)=\omega\left(f_{i}, f_{j}\right)=0 \forall i, j .
$$

Pick any $T \in \operatorname{End}_{\mathbb{R}}(V)$, and write $T$ with respect to this basis in block form as follows:

$$
T=\left(\begin{array}{cc}
A & B \\
C & D
\end{array}\right)
$$

Then (7.1) is equivalent to

$$
\left(\begin{array}{cc}
0 & I \\
-I & 0
\end{array}\right) \cdot\left(\begin{array}{cc}
A & B \\
C & D
\end{array}\right)=\left(\begin{array}{cc}
A^{t} & C^{t} \\
B^{t} & D^{t}
\end{array}\right) \cdot\left(\begin{array}{cc}
0 & I \\
-I & 0
\end{array}\right)
$$


where $I$ is the $n \times n$ identity matrix, and superscript $t$ denotes the transpose. The last equality can be rewritten as

$$
D=A^{t}, \quad B^{t}=-B, \quad C^{t}=-C .
$$

In particular, if $n$ is even, we can choose $A$ so that $A^{2}=-1$; taking $B=C=$ 0 , we get an example of an operator $T$ satisfying (7.1) such that $T^{2}=-1$.

Observation. This provides an example of a situation where a $B$-field transform of a symplectic GCS is also $\beta$-complex.

Remark 7.3. One of the reasons why this examples is interesting is that it provides an example of a continuous deformation of a symplectic structure into a complex structure through GC structures. Indeed, it suffices to observe that if $\mathcal{J}$ is a GCS on $V$ and $B$ (resp., $\beta$ ) is a $B$-field (resp., $\beta$-field), and for every $t \in[0,1]$ we define $\mathcal{J}_{t}$ to be the transform of $\mathcal{J}$ by $t \cdot B$ (resp., $t \cdot \beta$ ), then $\mathcal{J}_{t}$ depends continuously on $t$, and $\mathcal{J}_{0}=\mathcal{J}$, whereas $\mathcal{J}_{1}$ is the transform of $\mathcal{J}$ by $B$ (resp., $\beta$ ).

Remark 7.4. As a by-product of our discussion, we also obtain an example of a subspace of a $B$-symplectic vector space which satisfies the graph condition with respect to some GC structure, but is not a GC subspace in the sense of Definition 3.2. For example, if $W$ is the subspace of $V$ spanned by $e_{1}, \ldots, e_{n}$, then $W$ is invariant under the operator $T$ defined above, so $\left.T\right|_{W}$ gives a complex structure on $W$. It is then easy to see (using Proposition 4.11) that $W$ satisfies the graph condition with respect to this complex structure. However, $W$ is obviously not a GC subspace of $V$ (cf. Proposition $7.1)$, since $\left.\omega\right|_{W} \equiv 0$.

Now let us pass to the global case. Thus $\omega$ is a symplectic form on a real manifold $M$, and $T$ is an $\mathbb{R}$-linear bundle endomorphism of $T M$ satisfying (7.1) for all sections $u, v$ of $T M$. We suppose that if $B$ is the two-form on $M$ corresponding to $T$, then the $B$-field transform of the GCS defined by $\omega$ is integrable. Let $E \subset T_{\mathbb{C}} M \oplus T_{\mathbb{C}}^{*} M$ denote the maximally isotropic subbundle corresponding to this transform. It is clear that $E \cap T_{\mathbb{C}} M$ is the $+i$-eigensheaf of $T$ on $T_{\mathbb{C}} M$. Clearly this sheaf is closed under the Lie bracket of vector fields (because the restriction of the Courant bracket to $T_{\mathbb{C}} M$ is the usual Lie bracket). The same applies to $\bar{E} \cap T_{\mathbb{C}} M$. Now $\left(E \cap T_{\mathbb{C}} M\right) \oplus\left(\bar{E} \cap T_{\mathbb{C}} M\right)$ is the complexification of the subsheaf $\operatorname{Ker}\left(1+T^{2}\right) \subseteq T M$, whence the latter subsheaf is integrable.

There exists a dense open set $U \subseteq M$ on which $1+T^{2}$ has constant rank. On this set, the three sheaves considered above become subbundles of $T_{\mathbb{C}} M$. Thus we obtain

Corollary 7.5. There exists a natural foliation of $U$, corresponding to the integrable smooth distribution $\operatorname{Ker}\left(1+T^{2}\right)$, whose leaves acquire a natural complex structure. 
This corollary is a global analogue of a special case of Theorem 3.11(b).

7.5. Some counterexamples. We start by giving an example of a $B$ symplectic vector space $V$ and a GC subspace $W \subset V$ such that $V / W$ is not a GC quotient of $V$.

Example 7.6. Let $V$ be a real vector space with basis $p_{1}, q_{1}, p_{2}, q_{2}$, and let $a_{1}, b_{1}, a_{2}, b_{2}$ be the dual basis of $V^{*}$. Define $\omega=a_{1} \wedge b_{1}+a_{2} \wedge b_{2}$, a symplectic form on $V$, and $B=a_{1} \wedge a_{2}-b_{1} \wedge b_{2}$, a $B$-field on $V$. Let $W$ be the plane in $V$ spanned by $p_{1}$ and $q_{1}$. Then $W$ is a GC subspace of $V$, but $V / W$ is not a GC quotient of $V$.

Indeed, it is clear that $W$ is a GC subspace of $V$, because $\left.\omega\right|_{W}$ is nondegenerate. Let $\pi: V \rightarrow V / W$ denote the quotient map, and let $\eta: \operatorname{Ann}(W) \rightarrow$ $(V / W)^{*}$ be the natural isomorphism. It follows from $\S 7.1$ and (3.2) that

$$
E_{V / W}=\left\{\left(\pi(v), \eta\left(\iota_{v}(B-i \omega)\right)\right) \mid \iota_{v}(B-i \omega) \in \operatorname{Ann}\left(W_{\mathbb{C}}\right)\right\} .
$$

Consider the element $v=p_{1}+i q_{2}$. It is easy to compute that $\iota_{v}(B-i \omega)=0$. Thus, $(\pi(v), 0) \in E_{V / W}$. But since $p_{1} \in W$, we have $\overline{\pi(v)}=\overline{\pi\left(i q_{2}\right)}=$ $\pi\left(-i q_{2}\right)=-\pi(v)$, whence we also have $(\pi(v), 0) \in \bar{E}_{V / W}$. This implies that $E_{V / W} \cap \bar{E}_{V / W} \neq(0)$, so $V / W$ is not a GC quotient of $V$.

Now we discuss a more complicated example.

Example 7.7. There exists a $B$-symplectic vector space $V$ with the following property. If $C$ is the minimal subspace of $V$ such that $V / C$ is a $\beta$-symplectic quotient of $V$ (cf. Theorem 3.11), then $C$ is not a GC subspace of $V$. Hence, by duality, we see that there exists a $\beta$-symplectic vector space $V$ such that, if $S$ is the maximal $B$-symplectic subspace of $V$, then $V / S$ is not a GC quotient of $V$.

To find an example of such a $V$, we use the theory developed in $\S 7.4$. We suppose that $V$ has a distinguished basis $\left\{e_{i}, f_{i}\right\}_{i=1}^{n}$, and is equipped with a symplectic form $\omega$ defined by (7.2). We consider an operator $T$ on $V$, represented by the matrix (7.3) (where $A, B, C, D$ are to be chosen later). It is clear that with this notation, the subspace $C \subset V$ is nothing but the kernel of $1+T^{2}$. Now we note that the equation (7.1) implies that $\operatorname{Ker}\left(1+T^{2}\right)$ is orthogonal to $\operatorname{Im}\left(1+T^{2}\right)$ with respect to $\omega$. Thus, if $\operatorname{Ker}\left(1+T^{2}\right) \cap \operatorname{Im}\left(1+T^{2}\right) \neq(0)$, the restriction of $\omega$ to $\operatorname{Ker}\left(1+T^{2}\right)$ is degenerate, so that $C=\operatorname{Ker}\left(1+T^{2}\right)$ is not a GC subspace of $V$.

It remains to choose an arbitrary $n \times n$ matrix $A$ such that $\operatorname{Ker}\left(1+A^{2}\right) \cap$ $\operatorname{Im}\left(1+A^{2}\right) \neq(0)$; for instance, one can take

$$
A=\left(\begin{array}{cc}
J & I \\
0 & J
\end{array}\right)
$$


where

$$
J=\left(\begin{array}{cc}
0 & 1 \\
-1 & 0
\end{array}\right), \quad I=\left(\begin{array}{ll}
1 & 0 \\
0 & 1
\end{array}\right)
$$

so that

$$
1+A^{2}=\left(\begin{array}{cc}
0 & 2 J \\
0 & 0
\end{array}\right)
$$

is a nonzero nilpotent matrix. Setting $D=A^{t}, B=C=0$, we obtain an example of an operator $T$ of the form (7.3), satisfying (7.4), and such that $\operatorname{Ker}\left(1+T^{2}\right) \cap \operatorname{Im}\left(1+T^{2}\right) \neq(0)$.

Remark 7.8. We leave it to the reader to check that an example of the type presented above does not exist in the $B$-complex case. That is, if $V$ is a $B$-complex $\mathrm{GC}$ vector space, and $C$ is the minimal subspace such that $V / C$ is a $\beta$-symplectic GC quotient of $V$, then $C$ is a GC subspace of $V$, and the induced structure on $C$ is complex.

\section{Proofs}

8.1. Proofs for section 2. We start by proving Proposition 2.1. A bijection between the collection of $\mathcal{J} \in \operatorname{Aut}_{\mathbb{R}}\left(V \oplus V^{*}\right)$ and the collection of $E \subset V_{\mathbb{C}} \oplus V_{\mathbb{C}}^{*}$ is obtained by associating to $\mathcal{J}$ its $+i$-eigenspace in $V_{\mathbb{C}} \oplus V_{\mathbb{C}}^{*}$. [Conversely, if we start with $E$, we consider the $\mathbb{C}$-linear automorphism of $V_{\mathbb{C}} \oplus V_{\mathbb{C}}^{*}$ defined as multiplication by $+i$ on $E$ and multiplication by $-i$ on $\bar{E}$; it is straightforward to check that this automorphism is induced by an $\mathbb{R}$-linear automorphism of $V \oplus V^{*}$.] On the other hand, a bijection between the collection of pure spinors $\phi \in \Lambda^{\bullet} V_{\mathbb{C}}^{*}$ and the collection of $E$ is obtained by associating to $\phi$ its annihilator in $V_{\mathbb{C}} \oplus V_{\mathbb{C}}^{*}$ with respect to the Clifford action (cf. §2.2). The fact that this is a bijection follows from parts (2.5) and (2.5) of Theorem 2.5.

For the subsequent arguments, we will need an explicit description of the inverse of the bijection between $\phi$ 's and $E$ 's. Suppose that $\phi \in \wedge^{\bullet} V_{\mathbb{C}}^{*}$ is a pure spinor, written in the form of Theorem 2.5(2.5): $\phi=\exp (u) \wedge f_{1} \wedge$ $\cdots \wedge f_{k}$. Let $\Phi$ denote the span of $f_{1}, \ldots, f_{k}$; this is a $k$-dimensional complex subspace of $V_{\mathbb{C}}^{*}$. We claim that

$$
E_{\phi}:=\left\{v-\iota_{v}(u)+f \mid v \in \operatorname{Ann}(\Phi), f \in \Phi\right\}
$$

is the maximally isotropic subspace corresponding to $\phi$. Indeed, it is obvious that $\operatorname{dim}_{\mathbb{C}} E_{\phi}=\operatorname{dim}_{\mathbb{C}} V_{\mathbb{C}}$, so it suffices to see that all elements of $E$ annihilate $\phi$. By definition of the Clifford action, we have $f \cdot \phi=f \wedge \phi=0$ for all $f \in \Phi$. Next, if $v \in \operatorname{Ann}(\Phi)$, then, using the fact that $\iota_{v}$ is a superderivation of the algebra $\wedge^{\bullet} V_{\mathbb{C}}^{*}$, we find that

$\iota_{v}(\phi)=\iota_{v}(\exp (u)) \wedge f_{1} \wedge \cdots \wedge f_{k}=\iota_{v}(u) \wedge \exp (u) \wedge f_{1} \wedge \cdots \wedge f_{k}=\iota_{v}(u) \wedge \phi$, whence also $\left(v-\iota_{v}(u)\right) \cdot \phi=\iota_{v}(\phi)-\iota_{v}(u) \wedge \phi=0$. We can now give the 
Proof of Proposition 2.8. Let $\mathcal{B}$ be the orthogonal automorphism of $V \oplus$ $V^{*}$ corresponding to $B$, as defined in $\S 2.4$. Explicitly, $\mathcal{B}$ acts as follows: $\mathcal{B}(v+f)=v+\iota_{v}(B)+f$. Now let us write $\phi$ in the canonical form: $\phi=$ $\exp (u) \wedge f_{1} \wedge \cdots \wedge f_{k}$. It is clear from Theorem 2.5(2.5) that $\psi:=\exp (-B) \wedge \phi$ is also a pure spinor. Using (8.1), we immediately find that $E_{\psi}=\mathcal{B} \cdot E_{\phi}$, which proves the proposition.

Before we proceed, we list some useful formulas. If $V$ is a real vector space and $\mathcal{J}$ is an $\mathbb{R}$-linear automorphism of $V \oplus V^{*}$, written, as usual, in matrix form:

$$
\mathcal{J}=\left(\begin{array}{cc}
\mathcal{J}_{1} & \mathcal{J}_{2} \\
\mathcal{J}_{3} & \mathcal{J}_{4}
\end{array}\right)
$$

then the conditions that $\mathcal{J}$ preserves the pairing $\langle\cdot, \cdot\rangle$ and satisfies $\mathcal{J}^{2}=-1$ are equivalent to the following collection of equations:

$$
\begin{aligned}
& \mathcal{J}_{1}^{2}+\mathcal{J}_{2} \mathcal{J}_{3}=-1 \\
& \mathcal{J}_{1} \mathcal{J}_{2}+\mathcal{J}_{2} \mathcal{J}_{4}=0 \\
& \mathcal{J}_{3} \mathcal{J}_{1}+\mathcal{J}_{4} \mathcal{J}_{3}=0 \\
& \mathcal{J}_{4}^{2}+\mathcal{J}_{3} \mathcal{J}_{2}=-1 \\
& \mathcal{J}_{4}=-\mathcal{J}_{1}^{*} \\
& \mathcal{J}_{2}^{*}=-\mathcal{J}_{2} \\
& \mathcal{J}_{3}^{*}=-\mathcal{J}_{3} .
\end{aligned}
$$

The verification of this claim is straightforward. It is used in the proof below.

Proof of Proposition 2.11. We first note that in parts (a) and (c), it is enough to prove the equivalence of the given statements involving $B$-field transforms, because then the analogous result for $\beta$-field transforms follows formally with the aid of duality ( $\S 2.3$ and Remark 2.9). Moreover, part (b) is immediate from the explicit formulas of $\S 7.1$. For part (d), note that if $\mathcal{J}_{1}=0$, then the equations 8.2-8.8 imply that $\mathcal{J}_{4}=0$ and $\mathcal{J}_{2}=-\mathcal{J}_{3}^{-1}$, with $\mathcal{J}_{2}^{*}=-\mathcal{J}_{2}$. Hence, taking $\omega=\mathcal{J}_{3}$, we see from Definition 2.10 that $E$ is symplectic.

The equivalences

$$
\rho(E) \cap \rho(\bar{E})=(0) \quad \Longleftrightarrow \quad V_{\mathbb{C}}^{*}=\left(V_{\mathbb{C}}^{*} \cap E\right)+\left(V_{\mathbb{C}}^{*} \cap \bar{E}\right) \quad \Longleftrightarrow \mathcal{J}_{2}=0
$$

and

$$
E \cap V_{\mathbb{C}}^{*}=(0) \Longleftrightarrow \rho(E)=V_{\mathbb{C}} \Longleftrightarrow \mathcal{J}_{2} \text { is an isomorphism }
$$

are immediate.

Now if $E$ is $B$-complex (resp., $B$-symplectic), it is clear from $\S 7.1$ that $\mathcal{J}_{2}=0$ (resp., $\mathcal{J}_{2}$ is an isomorphism). Conversely, suppose that $\mathcal{J}_{2}=0$. Then it is easy to check, using the formulas (8.2)-(8.8), that $\mathcal{J}_{1}^{2}=-1$ and $\mathcal{J}_{4}=-\mathcal{J}_{1}^{*}$. We put $J=\mathcal{J}_{1}$; thus $J$ is a complex structure on $V$. If we let 
$B=-(1 / 2) \cdot \mathcal{J}_{3} \cdot \mathcal{J}_{1}$, it is straightforward to check that $B$ is skew-symmetric and $\mathcal{J}_{3}=B \mathcal{J}_{1}+\mathcal{J}_{1}^{*} B$. Thus $E$ is $B$-complex.

Finally, suppose that $\mathcal{J}_{2}$ is an isomorphism. We put $\omega=-\mathcal{J}_{2}^{-1}$ and $B=-\mathcal{J}_{2}^{-1} \mathcal{J}_{1}$. Again, it is straightforward to verify, using (8.2)-(8.8), that $\omega^{*}=-\omega, B^{*}=-B$, and that $\mathcal{J}_{1}=\omega^{-1} B, \mathcal{J}_{2}=-\omega^{-1}, \mathcal{J}_{3}=\omega+B \omega^{-1} B$, $\mathcal{J}_{4}=-B \omega^{-1}$. Thus $E$ is $B$-symplectic, completing the proof.

8.2. Proofs for section 3. We begin with the following lemma, whose proof is completely straightforward from the definitions and is therefore omitted. Let $V$ be a real vector space, let $E \subseteq V_{\mathbb{C}} \oplus V_{\mathbb{C}}^{*}$ be a maximally isotropic subspace, and let $W \subseteq V$ be a subspace. Write $\tau: V \oplus V^{*} \rightarrow$ $V^{*} \oplus V, \tau_{W}:(V / W) \oplus(V / W)^{*} \rightarrow(V / W)^{*} \oplus(V / W)$ for the isomorphisms interchanging the summands. Define $E_{W}$ and $E_{V / W}$ by the formulas (3.1) and (3.2), respectively. Note that $\tau(E)$ is a maximally isotropic subspace of $V_{\mathbb{C}}^{*} \oplus V_{\mathbb{C}}$ and $\operatorname{Ann}(W)$ is a real subspace of $V^{*}$, so it makes sense to consider $\tau(E)_{\operatorname{Ann}(W)}$. Finally, we identify $\operatorname{Ann}(W)$ with $V / W$ in the obvious way.

Lemma 8.1. With this identification, we have

$$
\tau(E)_{\mathrm{Ann}(W)}=\tau_{W}\left(E_{V / W}\right) .
$$

Our next result is a computation.

Lemma 8.2. Let $V$ be a real vector space with a $G C$ structure given by $E \subseteq$ $V_{\mathbb{C}} \oplus V_{\mathbb{C}}^{*}$. Let $W \subseteq V$ be a subspace, and define $E_{W}, E_{V / W}$ by (3.1) and (3.2), respectively. Then $\operatorname{dim}_{\mathbb{C}} E_{W}=\operatorname{dim}_{\mathbb{R}} W$ and $\operatorname{dim}_{\mathbb{C}} E_{V / W}=\operatorname{dim}_{\mathbb{R}}(V / W)$.

Proof. Note that the second equality is equivalent to the first, in view of Lemma 8.1. Thus we will only prove the first equality. It is clear that $\rho\left(E_{W}\right)=\rho(E) \cap W_{\mathbb{C}}$, so projection to $W_{\mathbb{C}}$ realizes $E_{W}$ as an extension

$$
0 \longrightarrow K \longrightarrow E_{W} \longrightarrow \rho(E) \cap W_{\mathbb{C}} \longrightarrow 0,
$$

where $K$, in turn, fits into a short exact sequence

$$
0 \longrightarrow E \cap \operatorname{Ann}\left(W_{\mathbb{C}}\right) \longrightarrow E \cap V_{\mathbb{C}}^{*} \longrightarrow K \longrightarrow 0
$$

(the last nonzero arrow is given by restriction to $W$ ). Thanks to the fact that $E$ is maximally isotropic, we have $E \cap V_{\mathbb{C}}^{*}=\operatorname{Ann}(\rho(E))$. Thus

$$
E \cap \operatorname{Ann}\left(W_{\mathbb{C}}\right)=E \cap V_{\mathbb{C}}^{*} \cap \operatorname{Ann}\left(W_{\mathbb{C}}\right)=\operatorname{Ann}\left(\rho(E)+W_{\mathbb{C}}\right),
$$

which implies that

$$
\begin{aligned}
\operatorname{dim}_{\mathbb{C}}\left(E \cap \operatorname{Ann}\left(W_{\mathbb{C}}\right)\right)= & \operatorname{dim}_{\mathbb{C}} V_{\mathbb{C}}-\operatorname{dim}_{\mathbb{C}}\left(\rho(E)+W_{\mathbb{C}}\right) \\
= & \operatorname{dim}_{\mathbb{R}} V-\operatorname{dim}_{\mathbb{R}} W-\operatorname{dim}_{\mathbb{C}} \rho(E) \\
& +\operatorname{dim}_{\mathbb{C}}\left(\rho(E) \cap W_{\mathbb{C}}\right) .
\end{aligned}
$$


Therefore

$$
\begin{aligned}
\operatorname{dim}_{\mathbb{C}}\left(E_{W}\right) & =\operatorname{dim}_{\mathbb{C}}\left(\rho(E) \cap W_{\mathbb{C}}\right)+\operatorname{dim}_{\mathbb{C}} K \\
& =\operatorname{dim}_{\mathbb{C}}\left(\rho(E) \cap W_{\mathbb{C}}\right)+\operatorname{dim}_{\mathbb{C}}\left(E \cap V_{\mathbb{C}}^{*}\right)-\operatorname{dim}_{\mathbb{C}}\left(E \cap \operatorname{Ann}\left(W_{\mathbb{C}}\right)\right) \\
& =\operatorname{dim}_{\mathbb{C}}\left(E \cap V_{\mathbb{C}}^{*}\right)-\operatorname{dim}_{\mathbb{R}} V+\operatorname{dim}_{\mathbb{R}} W+\operatorname{dim}_{\mathbb{C}} \rho(E)=\operatorname{dim}_{\mathbb{R}} W,
\end{aligned}
$$

completing the proof.

Proof of Proposition 3.5. The product $f_{1} \wedge \cdots \wedge f_{k}$ depends (up to a sign) only on the span of the $f_{i}$ 's, and we know from Theorem $2.5(2.5)$ that the span must equal to $E \cap V_{\mathbb{C}}^{*}$. Thus, we are free to choose the $f_{i}$ 's to be any basis of this space. Since $E$ is maximally isotropic, we have $E \cap V_{\mathbb{C}}^{*}=\operatorname{Ann}(\rho(E))$, and now the existence of the basis we are looking for follows from the short exact sequence

$$
0 \longrightarrow \operatorname{Ann}\left(\rho(E)+W_{\mathbb{C}}\right) \longrightarrow \operatorname{Ann}(\rho(E)) \stackrel{j^{*}}{\longrightarrow} \operatorname{Ann}\left(\rho(E) \cap W_{\mathbb{C}}\right) \longrightarrow 0,
$$

where we view the last space as a subspace in $W_{\mathbb{C}}^{*}$. [The surjectivity follows, for instance, from dimension counting.]

Now define $\phi_{W}$ by the formula given in Proposition 3.5. Theorem 2.5(2.5) implies that $\phi_{W}$ is a pure spinor, and (8.1) shows that

$$
E=E_{\phi}=\left\{v-\iota_{v}(u)+f \mid v \in \rho(E), f \in E \cap V_{\mathbb{C}}^{*}=\operatorname{Ann}(\rho(E))\right\},
$$

and the maximally isotropic subspace corresponding to $\phi_{W}$ is

$$
E_{\phi_{W}}=\left\{w-\iota_{w}(u)+g \mid w \in \operatorname{Ann}\left(\Phi_{W}\right), g \in \Phi_{W}\right\},
$$

where $\Phi_{W}$ is the span of $j^{*}\left(f_{1}\right), \ldots, j^{*}\left(f_{l}\right)$, i.e., by construction, $\Phi_{W}=$ $\operatorname{Ann}\left(\rho(E) \cap W_{\mathbb{C}}\right) \subseteq W_{\mathbb{C}}^{*}$. It is then clear that $E_{W} \subseteq E_{\phi_{W}}$, and equality holds for reasons of dimension.

We now come to the proofs of Theorems 3.10 and 3.11. Let $V$ be an arbitrary GC vector space; we let $\mathcal{J} \in \operatorname{Aut}_{\mathbb{R}}\left(V \oplus V^{*}\right)$ denote the corresponding orthogonal automorphism, and $E \subset V_{\mathbb{C}} \oplus V_{\mathbb{C}}^{*}$ the corresponding subspace. Let $C$ be the real subspace of $V$ such that $C_{\mathbb{C}}=\left(E \cap V_{\mathbb{C}}\right) \oplus\left(\bar{E} \cap V_{\mathbb{C}}\right)$. Then $C$ is obviously invariant under $\mathcal{J}$, so $\left.\mathcal{J}\right|_{C}$ gives a canonical complex structure on $C$. It is clear from Proposition 4.11(a) that $C$, with this structure, satisfies the graph condition. Furthermore, if $C$ is a GC subspace of $V$, then Proposition 4.11(c) implies that the induced structure on $C$ is $\beta$-complex, and the underlying complex structure coincides with $\left.\mathcal{J}\right|_{C}$.

On the other hand, there exists a real subspace $S \subseteq V$ such that $S_{\mathbb{C}}=$ $\rho(E) \cap \rho(\bar{E})$. It is easy to check that $S$ is a GC subspace of $V$ (also see the proof of Theorem 3.10 below). Moreover, Proposition 2.11(c) implies that $S$ is the maximal GC subspace of $V$ such that the induced structure is $B$ symplectic. Next, $S_{\mathbb{C}}=\operatorname{Ann}\left(\left(E \cap V_{\mathbb{C}}^{*}\right) \oplus\left(\bar{E} \cap V_{\mathbb{C}}^{*}\right)\right)$. If $\tilde{C}$ is the real subspace of $V^{*}$ such that $\tilde{C}_{\mathbb{C}}=\left(E \cap V_{\mathbb{C}}^{*}\right) \oplus\left(\bar{E} \cap V_{\mathbb{C}}^{*}\right)$, so that $\tilde{C}=\operatorname{Ann}(S)$, then, applying the argument of the previous paragraph to the dual structure $\tau(E)$, we see 
that $V / S \cong \tilde{C}^{*}$ acquires a natural complex structure, and hence so does $\tilde{C}$. Furthermore, if $V / S$ is a GC quotient of $V$, then by Proposition 3.9, $\tilde{C}$ is a GC subspace of $V^{*}$, and now the argument of the previous paragraph shows that $\tilde{C}$ is $\beta$-complex, whence $V / S$ is $B$-complex. On the other hand, if we return to the situation of the previous paragraph and use our results about $S$, we see that $V / C$ is always a GC quotient of $V$, and the induced structure is $\beta$-symplectic; and, moreover, $C$ is the smallest subspace of $V$ with this property. This completes the proof of Theorem 3.11.

To prove Theorem 3.10 , let us begin by constructing the maximal $B$ symplectic GC subspace $S \subseteq V$ as in the previous paragraph. This characterization of $S$ shows that $S$ does not change if we make a $B$-field transform of the GCS on $V$ (and neither does the result of the theorem). Therefore, we assume, from the beginning, that the pure spinor defining our GCS has the form $\phi=\exp (i \Omega) \wedge f_{1} \wedge \cdots \wedge f_{k}$, where $\Omega \in \wedge^{2} V^{*}$ is a real 2-form. We know that $f_{1}, \ldots, f_{k}$ form a basis for $E \cap V_{\mathbb{C}}^{*}=\operatorname{Ann}(\rho(E))$, and hence $f_{1}, \ldots, f_{k}, \bar{f}_{1}, \ldots, \bar{f}_{k}$ form a basis for $\operatorname{Ann}\left(S_{\mathbb{C}}\right)$. By Theorem 2.5(2.5), we have, up to a nonzero scalar multiple,

$$
0 \neq\langle\phi, \bar{\phi}\rangle_{M}=\Omega^{p} \wedge f_{1} \wedge \cdots \wedge f_{k} \wedge \bar{f}_{1} \wedge \cdots \wedge \bar{f}_{k}
$$

where $p=\operatorname{dim}_{\mathbb{R}} S$. This implies that the restriction of $\Omega$ to $S$ must be nondegenerate; write $\omega=\left.\Omega\right|_{S}$. Basic linear algebra shows that if $W$ is the orthogonal complement to $S$ with respect $\Omega$, then $V=S \oplus W$ (direct sum of vector spaces). Let $\pi_{S}: V \rightarrow S, \pi_{W}: V \rightarrow W$ be the two projections, and let $g_{j}=\left.f_{j}\right|_{W_{\mathbb{C}}}(1 \leq j \leq k), u=\left.i \Omega\right|_{W_{\mathbb{C}}}$. Note that the $g_{j}, \bar{g}_{j}$ form a basis of $W_{\mathbb{C}}^{*}$. Now Proposition 3.5 implies that the pure spinors corresponding to $S$ and $W$ are given by

$$
\phi_{S}=\exp (i \omega) \quad \text { and } \quad \phi_{W}=\exp (u) \wedge f_{1} \wedge \cdots \wedge f_{k},
$$

respectively. Then Theorem 2.5(2.5) gives (up to a nonzero scalar multiple)

$$
\left\langle\phi_{S}, \bar{\phi}_{S}\right\rangle_{M}=\omega^{p} \quad \text { and } \quad\left\langle\phi_{W}, \bar{\phi}_{W}\right\rangle_{M}=g_{1} \wedge \cdots \wedge g_{k} \wedge \bar{g}_{1} \wedge \cdots \wedge \bar{g}_{k} ;
$$

in particular, both $S$ and $W$ are GC subspaces of $V$. Moreover, by construction, $W_{\mathbb{C}}^{*}=\left(E_{W} \cap W_{\mathbb{C}}^{*}\right) \oplus\left(\bar{E}_{W} \cap W_{\mathbb{C}}^{*}\right)$, whence $W$ is $B$-complex by Proposition 2.11(a). Finally, since the $f_{j}$ annihilate $S_{\mathbb{C}}$, and since $S$ is orthogonal to $W$ with respect to $\Omega$, it is easy to see that

$$
\phi=\pi_{S}^{*}\left(\phi_{S}\right) \wedge \pi_{W}^{*}\left(\phi_{W}\right),
$$

which shows that $V=S \oplus W$ as GC vector spaces, and completes the proof of Theorem 3.10. 
8.3. Proofs for section 4. First let us briefly discuss the proof of Proposition 4.8. It is obvious that $N \oplus \operatorname{Ann}(N)$ is the orthogonal complement of $W \oplus \operatorname{Ann}(W)$ with respect to our standard pairing $\langle\cdot, \cdot\rangle$; since $\mathcal{J}$ is orthogonal with respect to this pairing, we see that $N \oplus \operatorname{Ann}(W)$ is invariant under $\mathcal{J}$. Now let $\psi: W \oplus \operatorname{Ann}(N) \rightarrow W \oplus W^{*}$ and $\psi^{\prime}: N \oplus \operatorname{Ann}(W) \rightarrow N \oplus N^{*}$ be the natural isomorphisms. Invariance under $\mathcal{J}$ implies that

$$
W_{\mathbb{C}} \oplus \operatorname{Ann}\left(N_{\mathbb{C}}\right)=\left(E \cap\left(W_{\mathbb{C}} \oplus \operatorname{Ann}\left(N_{\mathbb{C}}\right)\right)\right) \oplus\left(\bar{E} \cap\left(W_{\mathbb{C}} \oplus \operatorname{Ann}\left(N_{\mathbb{C}}\right)\right)\right) ;
$$

on the other hand, we have (by the definition of $E_{W}$ )

$$
\psi\left(E \cap\left(W_{\mathbb{C}} \oplus \operatorname{Ann}\left(N_{\mathbb{C}}\right)\right)\right) \subseteq E_{W},
$$

and similarly for $\bar{E}$. Comparing the dimensions, we see that equality in the formula above holds. Since $\psi$ is an isomorphism, it follows that $E_{W} \cap \bar{E}_{W}=$ (0). The rest is obvious. We can now give the

Proofs of Propositions 4.9-4.11. Consider a real vector space $V$ and a subspace $W \subseteq V$, and suppose that $V$ and $W$ are equipped with GC structures $\mathcal{J}$ and $\mathcal{K}$, respectively, written in the usual form as

$$
\mathcal{J}=\left(\begin{array}{cc}
\mathcal{J}_{1} & \mathcal{J}_{2} \\
\mathcal{J}_{3} & \mathcal{J}_{4}
\end{array}\right) \text { and } \mathcal{K}=\left(\begin{array}{cc}
\mathcal{K}_{1} & \mathcal{K}_{2} \\
\mathcal{K}_{3} & \mathcal{K}_{4}
\end{array}\right)
$$

The graph of the inclusion $W \hookrightarrow V$ is the subset $\Gamma \subset W \oplus V$ consisting of all pairs $(w, w)$ with $w \in W$, while $\operatorname{Ann}(\Gamma)$ is the subset of $W^{*} \oplus V^{*}$ consisting of all pairs $(f, g)$ with $f=-\left.g\right|_{W}$. Let $\mathcal{J}_{t p}$ denote the twisted product structure on $W \oplus V$; thus $\Gamma$ is a generalized isotropic subspace of $W \oplus V$ with respect to this structure if and only if $\mathcal{J}_{t p}(\Gamma) \subseteq \Gamma \oplus \operatorname{Ann}(\Gamma)$. Explicitly, if $w \in W$, so that $(w, w) \in \Gamma$, then

$$
\mathcal{J}_{t p}(w, w, 0,0)=\left(\mathcal{K}_{1}(w), \mathcal{J}_{1}(w),-\mathcal{K}_{3}(w), \mathcal{J}_{3}(w)\right)
$$

Therefore, $W$ (with the structure $\mathcal{K}$ ) satisfies the graph condition if and only if

$$
\mathcal{K}_{1}=\left.\mathcal{J}_{1}\right|_{W} \text { and } \mathcal{K}_{3}(w)=\left.\mathcal{J}_{3}(w)\right|_{W} \forall w \in W
$$

(in particular, the first condition implies that $W$ is invariant under $\mathcal{J}_{1}$ ). Note that (8.9) and (8.6) imply that $\mathcal{K}_{4}\left(\left.f\right|_{W}\right)=\left.\mathcal{J}_{4}(f)\right|_{W}$ for all $f \in V^{*}$. Thus, the requirement that $W$ satisfies the graph condition with respect to $\mathcal{K}$ determines $\mathcal{K}_{1}, \mathcal{K}_{3}$ and $\mathcal{K}_{4}$ uniquely. In view of Lemma 8.3, whose proof is given below, we see that the rest of Propositions 4.9-4.11 follow from the following

Claim. If $W$ is a GC subspace of $V$ which is invariant under $\mathcal{J}_{1}$, and $\mathcal{K}$ denotes the induced structure, then the conditions (8.9) are satisfied. 
To prove this claim, let $E$ be the $+i$-eigenspace of $\mathcal{J}$ in $V_{\mathbb{C}}$, and let $w \in W_{\mathbb{C}}$. We can write $w=e+e^{\prime}$, where $e \in E, e^{\prime} \in \bar{E}$. Then $\mathcal{J}(w)=$ $i \cdot\left(e-e^{\prime}\right)$. Now $\rho(e)+\rho\left(e^{\prime}\right)=w \in W_{\mathbb{C}}$, and $i \cdot\left(\rho(e)-\rho\left(e^{\prime}\right)\right)=\rho(\mathcal{J}(w))=$ $\mathcal{J}_{1}(w) \in W_{\mathbb{C}}$ by assumption, whence $\rho(e), \rho\left(e^{\prime}\right) \in W_{\mathbb{C}}$. Thus, in fact, $(w, 0)=\left(\rho(e),\left.\rho^{*}(e)\right|_{W_{\mathbb{C}}}\right)+\left(\rho\left(e^{\prime}\right),\left.\rho^{*}\left(e^{\prime}\right)\right|_{W_{\mathbb{C}}}\right)$, with $\left(\rho(e),\left.\rho^{*}(e)\right|_{W_{\mathbb{C}}}\right) \in E_{W}$ and $\left(\rho\left(e^{\prime}\right),\left.\rho^{*}\left(e^{\prime}\right)\right|_{W_{\mathbb{C}}}\right) \in \bar{E}_{W}$. Therefore

$$
\begin{aligned}
\left(\mathcal{K}_{1}(w), \mathcal{K}_{3}(w)\right) & =\mathcal{K}(w, 0)=i \cdot\left(\rho(e),\left.\rho^{*}(e)\right|_{W_{\mathbb{C}}}\right)-i \cdot\left(\rho\left(e^{\prime}\right),\left.\rho^{*}\left(e^{\prime}\right)\right|_{W_{\mathbb{C}}}\right) \\
& =\left(\mathcal{J}_{1}(w),\left.\mathcal{J}_{3}(w)\right|_{W_{\mathbb{C}}}\right),
\end{aligned}
$$

completing the proof.

Lemma 8.3. Let $\mathcal{J}$ and $\mathcal{J}^{\prime}$ be two $G C$ structures on a real vector space $V$ which have the following forms:

$$
\mathcal{J}=\left(\begin{array}{cc}
\mathcal{J}_{1} & \mathcal{J}_{2} \\
\mathcal{J}_{3} & \mathcal{J}_{4}
\end{array}\right) \text { and } \mathcal{J}^{\prime}=\left(\begin{array}{cc}
\mathcal{J}_{1} & \mathcal{J}_{2}^{\prime} \\
\mathcal{J}_{3} & \mathcal{J}_{4}
\end{array}\right)
$$

(thus, only the $(1,2)$-entries are different). Then there exists a $\beta \in \bigwedge^{2} V$ that transforms $\mathcal{J}$ into $\mathcal{J}^{\prime}$. Moreover, $\beta$ is unique if we impose the additional constraint that $\mathcal{J}_{1} \circ \beta: V^{*} \rightarrow V$ be skew-symmetric (here we view $\beta$ as a skew-symmetric linear map $V^{*} \rightarrow V$, as explained in $\left.\S 1.5\right)$.

Proof. All the computations below that we omit are straightforward if one uses (8.2)-(8.8). First one checks that the condition that $\beta \in \bigwedge^{2} V$ transforms $\mathcal{J}$ into $\mathcal{J}^{\prime}$ is equivalent to the following equations:

$$
\beta \mathcal{J}_{3}=\mathcal{J}_{3} \beta=0, \quad-\mathcal{J}_{1} \beta+\beta \mathcal{J}_{4}=\mathcal{J}_{2}^{\prime}-\mathcal{J}_{2} .
$$

Let $\mathcal{L}=(1 / 2) \cdot\left(\mathcal{J}_{2}-\mathcal{J}_{2}^{\prime}\right)$. If $\mathcal{J}_{1} \beta$ is to be skew-symmetric, then (since $\beta$ is also skew-symmetric) we must have $\beta \mathcal{J}_{4}=\beta^{*} \mathcal{J}_{1}^{*}=\left(\mathcal{J}_{1} \beta\right)^{*}=-\mathcal{J}_{1} \beta$, which forces $\mathcal{J}_{1} \beta=\mathcal{L}$. This equation and $\mathcal{J}_{3} \beta=0$ imply that $-\beta=$ $\left(\mathcal{J}_{1}^{2}+\mathcal{J}_{2} \mathcal{J}_{3}\right) \circ \beta=\mathcal{J}_{1} \mathcal{L}$, whence $\beta=(1 / 2) \cdot \mathcal{J}_{1} \circ\left(\mathcal{J}_{2}^{\prime}-\mathcal{J}_{2}\right)$, proving the uniqueness of $\beta$. Conversely, if we define $\beta$ by this formula, then one easily checks that $\beta^{*}=-\beta$, and the equations (8.10) are satisfied. This completes the proof.

\subsection{Proofs for section 5 .}

Proof of Proposition 5.7. Part (a) is immediate from the equality $d(f \cdot g)+$ $f \cdot Y+g \cdot X=f \cdot(d g+Y)+g \cdot(d f+X)$. For part (b), let $(f, X)$ be a section of $\mathcal{C O U}_{M}$ defined near a point $m \in M$. Suppose that $f(m) \neq 0$, then there exists an open set $U \ni m$ such that $(f, X)$ is defined on $U$, and is nonvanishing there. Then it is easy to check that $\left(f^{-1},-f^{-2} \cdot X\right) \in$ $\mathcal{C O U}_{M}(U)$, and $(f, X) \cdot\left(f^{-1},-f^{-2} \cdot X\right)=(1,0)$. Finally, part (c) follows 
from the definition of integrability in terms of the Courant bracket, and the easily verified identity

$$
[d f+X, d g+Y]_{\mathrm{cou}}=\frac{1}{2}(d(X(g))-d(Y(f)))+[X, Y]
$$

for all local sections $(f, X)$ and $(g, Y)$ of $C_{M}^{\infty} \oplus T_{\mathbb{C}} M$. We leave to the reader the straightforward but tedious verification that $\{\cdot, \cdot\}$ satisfies the Leibniz rule and the definition of a Poisson algebra; we remark that here the fact that $\bar{E}$ is isotropic is used in an essential way. (In other words, $\{\cdot, \cdot\}$ doesn't have these properties on the bigger sheaf $C_{M}^{\infty} \oplus T_{\mathbb{C}} M$.)

Proof of Theorem 5.13. The given map is clearly a ring homomorphism with respect to the ring operations defined in Proposition 5.7(a). Thus we only need to check that this map takes $\mathcal{C} \mathcal{O U}_{M}(U)$ into $\mathcal{C O U}_{S}(U \cap S)$. Let $j$ : $S \hookrightarrow M$ denote the inclusion map. We'll show, more generally, that if $X+\xi$ is a section of $\bar{E}$ over $U$, where $X \in \Gamma\left(U, T_{\mathbb{C}} M\right)$ and $\xi \in \Gamma\left(U, T_{\mathbb{C}}^{*} M\right)$, then $\pi\left(\left.X\right|_{S}\right)+j^{*}(\xi)$ is a section of $\bar{E}_{S}$ over $U \cap S$. To that end, write, on $U \cap S$ : $X=Y+Z, \xi=\eta+\zeta$, where $Y, Z, \eta$ and $\zeta$ are sections over $U \cap S$ of $T_{\mathbb{C}} S$, $N_{\mathbb{C}},\left.\operatorname{Ann}\left(N_{\mathbb{C}}\right) \subset T_{\mathbb{C}}^{*} M\right|_{S}$ and $\left.\operatorname{Ann}\left(T_{\mathbb{C}} S\right) \subset T_{\mathbb{C}}^{*} M\right|_{S}$, respectively. It is clear that $Y=\pi\left(\left.X\right|_{S}\right)$ and $\left.\eta\right|_{T_{\mathbb{C} S}}=j^{*}(\xi)$. Thus it remains to observe that $(Y, \eta)$ is a section of $\bar{E}$. This follows from the decomposition

$$
X+\xi=(Y+\eta)+(Z+\zeta) \in\left(T_{\mathbb{C}} S+\operatorname{Ann}\left(N_{\mathbb{C}}\right)\right) \oplus\left(N_{\mathbb{C}}+\operatorname{Ann}\left(T_{\mathbb{C}} S\right)\right)
$$

and the fact that each of the two direct summands on the right is $\mathcal{J}$ invariant, by the definition of a split submanifold.

\subsection{Proofs for section 6 .}

Proof of Theorem 6.1. The theorem follows immediately from its linear counterpart (Theorem 4 in the introduction), which we find convenient to restate as follows. Let $V$ be a real vector space, and let $\mathcal{J}, \mathcal{K}$ be two GC structures on $V$. If the diagonal $\Delta \subset V \oplus V$ is generalized Lagrangian with respect to the direct sum of the structures $\tilde{\mathcal{J}}$ and $\mathcal{K}$, then $\mathcal{J}=\mathcal{K}$.

Now $\operatorname{Ann}(\Delta)=\left\{(f,-f) \in V^{*} \oplus V^{*}\right\}$, and if $v \in V, f \in V^{*}$, then

$$
\begin{aligned}
(\tilde{\mathcal{J}} \oplus \mathcal{K})(v, v, f,-f)= & \left(\mathcal{J}_{1}(v)-\mathcal{J}_{2}(f), \mathcal{K}_{1}(v)-\mathcal{K}_{2}(f),\right. \\
& \left.-\mathcal{J}_{3}(v)+\mathcal{J}_{4}(f), \mathcal{K}_{3}(v)-\mathcal{K}_{4}(f)\right) .
\end{aligned}
$$

We see that $\Delta \oplus \operatorname{Ann}(\Delta)$ is invariant under $\tilde{\mathcal{J}} \oplus \mathcal{K}$ if and only if $\mathcal{J}_{1}=\mathcal{K}_{1}$, $\mathcal{J}_{2}=\mathcal{K}_{2}, \mathcal{J}_{3}=\mathcal{K}_{3}$ and $\mathcal{J}_{4}=\mathcal{K}_{4}$, completing the proof.

Proof of Theorem 6.3. In the following proof, we will always implicitly identify $(V \oplus W)^{*}$ with $V^{*} \oplus W^{*}$, and similarly for $W \oplus Z, V \oplus Z$. We begin with the following 
Claim. Let $(f, h) \in V^{*} \oplus Z^{*}$. Then $(f, h) \in \operatorname{Ann}(\Phi \circ \Gamma)$ if and only if there exists $g \in W^{*}$ such that $(f, g) \in \operatorname{Ann}(\Gamma)$ and $(-g, h) \in \operatorname{Ann}(\Phi)$.

Indeed, suppose first that there is a $g \in W^{*}$ with $(f, g) \in \operatorname{Ann}(\Gamma)$, $(-g, h) \in \operatorname{Ann}(\Phi)$. If $(v, z) \in \Phi \circ \Gamma$, then there exists $w \in W$ such that $(v, w) \in \Gamma$ and $(w, z) \in \Phi$. Then $f(v)+g(w)=0$ and $-g(w)+h(z)=0$, whence $f(v)+h(z)=0$. This shows that $(f, h) \in \operatorname{Ann}(\Phi \circ \Gamma)$.

Conversely, let $(f, h) \in \operatorname{Ann}(\Phi \circ \Gamma)$. Let $\Lambda$ (resp., $\Psi)$ denote the projection of $\Gamma$ (resp., $\Phi)$ onto $W$. Note that if $(v, 0) \in \Gamma$, then $(v, 0) \in \Phi \circ \Gamma$, so that $f(v)=-h(0)=0$; similarly, if $(0, z) \in \Phi$, then $(0, z) \in \Phi \circ \Gamma$, whence $h(z)=0$. This shows that we can define a linear functional $g_{1}$ on $\Lambda$ by the rule $g_{1}(w)=-f(v, w)$ for any $v \in V$ such that $(v, w) \in \Gamma$. Similarly, we can define a linear functional $g_{2}$ on $\Psi$ by the rule $g_{2}(w)=h(w, z)$ for any $z \in Z$ such that $(w, z) \in \Phi$. Then $g_{1}$ and $g_{2}$ agree on $\Lambda \cap \Psi$, because if $w \in \Lambda \cap \Psi$, then $(v, w) \in \Gamma$ and $(w, z) \in \Phi$ for some $v \in V, z \in Z$, whence $g_{1}(w)=-f(v)=h(z)=g_{2}(w)$. Thus, there exists a linear functional $g \in W^{*}$ such that $\left.g\right|_{\Lambda}=g_{1}$ and $\left.g\right|_{\Psi}=g_{2}$. By construction, we have $(f, g) \in \operatorname{Ann}(\Gamma)$ and $(g, h) \in \operatorname{Ann}(\Psi)$, proving the claim.

Now let us prove part (a) of the theorem. Let $\mathcal{J}_{V}, \mathcal{J}_{W}$ and $\mathcal{J}_{Z}$ denote the automorphisms defining the GC structures on $V, W$ and $Z$, respectively. We will write them in the usual $2 \times 2$ matrix form. Suppose that $\Gamma \subset V \oplus W$ and $\Phi \subset W \oplus Z$ are generalized isotropic subspace with respect to the twisted product structures. If $(v, z) \in \Phi \circ \Gamma$, then there exists $w \in W$ with $(v, w) \in \Gamma$ and $(w, z) \in \Phi$. Then we get

$$
\left(\tilde{\mathcal{J}}_{V} \oplus \mathcal{J}_{Z}\right)(v, z, 0,0)=\left(\mathcal{J}_{V 1}(v), \mathcal{J}_{Z 1}(z),-\mathcal{J}_{V 3}(v), \mathcal{J}_{Z 3}(z)\right) .
$$

We have

$$
\left(\tilde{\mathcal{J}}_{V} \oplus \mathcal{J}_{W}\right)(v, w, 0,0)=\left(\mathcal{J}_{V 1}(v), \mathcal{J}_{W 1}(w),-\mathcal{J}_{V 3}(v), \mathcal{J}_{W 3}(w)\right)
$$

and

$$
\left(\tilde{\mathcal{J}}_{W} \oplus \mathcal{J}_{Z}\right)(w, z, 0,0)=\left(\mathcal{J}_{W 1}(w), \mathcal{J}_{Z 1}(z),-\mathcal{J}_{W 3}(w), \mathcal{J}_{Z 3}(z)\right)
$$

whence

$$
\begin{aligned}
& \left(\mathcal{J}_{V 1}(v), \mathcal{J}_{W 1}(w)\right) \in \Gamma,\left(\mathcal{J}_{W 1}(w), \mathcal{J}_{Z 1}(z)\right) \in \Phi, \\
& \left(-\mathcal{J}_{V 3}(v), \mathcal{J}_{W 3}(w)\right) \in \operatorname{Ann}(\Gamma), \text { and }\left(-\mathcal{J}_{W 3}(w), \mathcal{J}_{Z 3}(z)\right) \in \operatorname{Ann}(\Phi),
\end{aligned}
$$

by assumption. This implies that $\left(\mathcal{J}_{V 1}(v), \mathcal{J}_{Z 1}(z)\right) \in \Phi \circ \Gamma$, and, according to the claim above, $\left(-\mathcal{J}_{V 3}(v), \mathcal{J}_{Z 3}(z)\right) \in \operatorname{Ann}(\Phi \circ \Gamma)$. This proves that $\Phi \circ \Gamma$ is generalized isotropic with respect to the twisted product structure on $V \oplus Z$.

For part (b), we assume that $\Gamma$ and $\Phi$ are generalized coisotropic with respect to the twisted product structures. Then we start with $(f, h) \in$ $\operatorname{Ann}(\Phi \circ \Gamma)$; according to the claim above, we can find a $g \in W^{*}$ such that 
$(f, g) \in \operatorname{Ann}(\Gamma)$ and $(-g, h) \in \operatorname{Ann}(\Phi)$. Then we proceed exactly as in the previous paragraph, and we deduce that

$$
\left(\tilde{\mathcal{J}}_{V} \oplus \mathcal{J}_{Z}\right)(0,0, f, h) \in(\Phi \circ \Gamma) \oplus \operatorname{Ann}(\Phi \circ \Gamma) .
$$

Finally, part (c) follows from parts (a) and (b).

\section{References}

[B] O. Ben-Bassat, Mirror Symmetry and Generalized Complex Manifolds, math.AG/ 0405303, submitted for publication.

[BR] H. Bursztyn and O. Radko, Gauge equivalence of Dirac structures and symplectic groupoids, Ann. Inst. Fourier (Grenoble) 53(1) (2003), 309-337, math.SG/0202099.

[CdS] A. Cannas da Silva, Lectures on Symplectic Geometry, Lecture Notes in Math., 1764, Springer-Verlag, Berlin, 2001.

[C] C. Chevalley, The Algebraic Theory of Spinors, part 2 of Vol. 2 of Chevalley's collected works, Springer-Verlag, 1996.

[Cou] T.J. Courant, Dirac manifolds, Trans. Amer. Math. Soc. 319 (1990), 631-661.

[Gua] M. Gualtieri, Generalized complex geometry, Ph.D. thesis, Oxford University, 2003, math.DG/0401221

[GS] V. Guillemin and S. Sternberg, Some problems in integral geometry and some related problems in microlocal analysis, Amer. J. Math. 101(4) (1979), 915-955.

[H1] N.J. Hitchin, The geometry of three-forms in six dimensions, J. Differential Geom. 55 (2000), 547-576, math.DG/0010054.

[H2] _ Generalized Calabi-Yau manifolds, Q. J. Math. 54(3) (2003), 281-308, math.DG/0209099.

[H3] Geometry with B-fields, Lectures at the 'Geometry, Topology, and Strings' miniprogram at KITP, Santa-Barbara, July 14 - August 8, 2003, available at: http://online.itp.ucsb.edu/online/mp03.

[Kap] A. Kapustin, Topological strings on noncommutative manifolds, preprint, 2003, hep-th/0310057.

[KO] A. Kapustin and D. Orlov, Vertex Algebras, Mirror Symmetry, And D-Branes: The Case Of Complex Tori, Comm. Math. Phys. 233 (2003), 79-136, hep-th/0010293.

[LWX] Z.-J. Liu, A. Weinstein and P. Xu, Manin triples for Lie bialgebroids, J. Differential Geom. 45(3) (1997), 547-574, dg-ga/9508013.

[NT] R. Nest and B. Tsygan, Deformations of symplectic Lie algebroids, deformations of holomorphic symplectic structures, and index theorems, Asian J. Math. 5(4) (2001), 599-635, math.QA/9906020.

[Roy] D. Roytenberg, Courant algebroids, derived brackets and even symplectic supermanifolds, math.DG/9910078.

[SW] P. Severa and A. Weinstein, Poisson geometry with a 3-form background, in 'Noncommutative geometry and string theory (Yokohama, 2001),' Progr. Theoret. Phys. Suppl. 144 (2001), 145-154, math.SG/0107133.

[TW] X. Tang and A. Weinstein, Quantization and Morita equivalence for constant Dirac structures on tori, preprint, 2003, math.QA/0305413. 
[V] I. Vaisman, Symplectic Geometry and Secondary Characteristic Classes, Birkhäuser Boston, 1987.

[W1] A. Weinstein, The symplectic "category", in 'Differential geometric methods in mathematical physics (Clausthal, 1980),' 45-51, Lecture Notes in Math., 905, Springer, Berlin-New York, 1982.

[W2] _ Symplectic geometry, Bull. Amer. Math. Soc. 5(1) (1981), 1-13.

DEPARTMENT OF MATHEMATICS

University of Pennsylvania

Philadelphia, PA 19104-6395

E-mail address: orenb@math.upenn.edu

Department of Mathematics

University of Chicago

Chicago, IL 60637

E-mail address: mitya@math.uchicago.edu 